\title{
Rational Design of a Fluorescent Microneedle Tattoo for Minimally Invasive
}

\section{Monitoring of Lymphatic Function}

Samuel Babity ${ }^{1}$, M.Sc.; Anna K. Polomska ${ }^{2}$, PhD; Frédéric Couture ${ }^{3}$, PhD; Mathias Bonmarin ${ }^{4}$, PhD; Daniel Fehr ${ }^{4}$, M.Sc., Michael Detmar ${ }^{2}$, M.D.; and Davide Brambilla ${ }^{1}, P h D$

${ }^{1}$ Faculté de Pharmacie, Université de Montréal, 2940 Chemin de Polytechnique, Montréal, Canada;

${ }^{2}$ Swiss Federal Institute of Technology (ETH Zürich), Institute of Pharmaceutical Sciences, VladimirPrelog-Weg 3, 8054 Zürich, Switzerland;

${ }^{3}$ TransBIOTech, 201 Monseigneur-Bourget, Lévis, Québec, G6V 6Z9, Canada;

${ }^{4}$ Zürich University of Applied Sciences, School of Engineering, Technikumstrasse 9, 8400 Winterthur, Switzerland.

Corresponding author: davide.brambilla@umontreal.ca 


\section{Abstract}

The monitoring of lymphatic drainage is of great importance, particularly in the context of the early detection and diagnosis of several diseases. Existing methods of imaging and monitoring lymphatic drainage can be costly and require trained personnel, posing problems for at-home or point-of-care monitoring. Recently, an alternative approach has been proposed, consisting of using microneedles to deliver a near-infrared (NIR) fluorescent tattoo to the skin, which can be monitored with traditional laboratory-based fluorescence detectors. In this work, we present further development of this approach, using a specifically designed NIR-fluorescent probe and rational optimization of microneedle properties and the spatial location of the NIR dye within the microneedles. Moreover, we demonstrate that this method is compatible with a custom-made portable fluorescence measurement device and able to discriminate between drainage and lack of drainage in vivo in rats.

Keywords: microneedles, monitoring, tattoo, diagnostics, lymphatics 


\section{Introduction}

The lymphatic system consists of a network of vessels and nodes that spread throughout the body, playing an essential role in tissue homeostasis, immune response, and dietary fat absorption in the intestine [1,2]. A variety of pathological conditions are associated with altered lymphatic function, including primary and secondary lymphedema [3,4], lipedema [5], venous insufficiencies [6], impaired wound healing [7], scleroderma [8], and psoriasis [9]. Among these, secondary lymphedema affects approximately 3 million people in the United States, and is characterized by an accumulation of interstitial fluid in the extremities due to impaired lymphatic drainage $[3,4]$. In the developed world, secondary lymphedema occurs most commonly as a complication of breast cancer treatment, primarily resulting from the operative excision of lymph nodes to limit potential metastasis $[10,11]$. Because of the significant impact to quality of life caused by this disorder, as well as the improved outcomes associated with rapid treatment, the early detection of secondary lymphedema is considered to be of critical importance [12].

In clinical practice, lymphedema is most often diagnosed based on clinical presentation, particularly changes in limb volume. While not part of routine diagnosis, medical imaging can be used to support initial clinical evaluation by providing a more quantitative assessment of lymphatic function. While lymphatic imaging techniques are less developed than those used for imaging the circulatory system, a variety of approaches are used, such as scintigraphy-based techniques (considered the goldstandard) [13], and more rarely, magnetic resonance [14] and computed tomography [15]. However, there remains significant drawbacks associated with the cost and complexity of these imaging strategies, which has led to the development of a new, simple, and affordable technique, namely lymphatic imaging using near-infrared (NIR) tracers [16]. This technique offers high sensitivity and spatial resolution, owing to the minimal tissue auto-fluorescence observed at NIR wavelengths $(>750 \mathrm{~nm})$, which enables a minimallyinvasive visualization of lymphatic vessels and assessment of their function [17]. This is notably limited to surface-level lymphatics, though NIR fluorescence does provide improved tissue penetration depth 
relative to other fluorescence imaging techniques $[18,19]$.

In addition to the detection of impaired lymphatic function, NIR fluorescence imaging has been studied extensively for the structural assessment of lymphatic vasculature [20-22]. In these initial studies, the most common NIR fluorescent dye used was indocyanine green (ICG) [23,24], a tricarbocyanine dye that can be excited around $780 \mathrm{~nm}$, with peak fluorescence emission around $813 \mathrm{~nm}$ [25]. Despite its advantages relative to other methods of lymphatic imaging, the delivery of ICG remains a painful procedure, requiring subcutaneous or intradermal injection to reach lymphatically vascularized tissues. Additionally, it has been reported that intradermal injection can lead to a localized increase in interstitial pressure within the dermis, potentially increasing the rate of ICG drainage and interfering with measurements $[26,27]$. A proposed solution to this issue has been the delivery of ICG using microneedles (MNs) [28]. Consisting of miniaturized needles with lengths below $1 \mathrm{~mm}$, often assembled into an array, MNs are long enough to pierce the skin's outer barrier — the epidermis — but short enough to avoid activating pain-sensing neurons found deeper in the tissue $[29,30]$. While initially proposed for the delivery of therapeutics, in recent years MNs have been increasingly studied for their potential diagnostic applications [31]. In a previous study, dissolving polymeric MNs were shown in mice to be able to effectively deliver ICG across the stratum corneum, allowing the visualization of lymphatic vessels [28]. Soluble polymeric MNs offer several advantages over other described classes (e.g. solid metal MNs or hollow silicon MNs), due to their simple manufacturing process, easy disposal, and the ability to incorporate a fluorescent dye directly into the structure of the MNs, allowing release upon dissolution in the skin [32-34].

In numerous works on lymphatic imaging, the NIR dye selected was ICG, likely based on its FDA-approved status and long history of clinical use, dating to 1959 [35]. While it remains the only currently clinically approved NIR tracer, ICG poses several limitations for use in vivo including its rapid degradation, poor solubility (resulting in fluorescence quenching), and low quantum yield [25,36,37]. Of particular note is the increase in quantum yield displayed by ICG upon binding to proteins in vivo, which 
could complicate rapid initial measurements of lymphatic drainage [38]. Further, studies have demonstrated that administration of ICG can result in acute impairment of lymphatic function, which could pose significant problems when using the dye to quantify lymphatic drainage $[39,40]$. Finally, as a small molecule, ICG has been previously shown to have limited selectivity for drainage via the lymphatic system and can also be drained through blood vessels, thus limiting the specificity and sensitivity of this imaging technique [41]. A potential solution to these issues lies in the use of PEGylated dye conjugates, which have been shown to promote selective lymphatic targeting in the context of drug delivery in vivo [42-44]. PEGylation increases the size of the dye molecule and has been shown to allow for greater lymphatic specificity; with the added benefit of improved solubility, due to the hydrophilic nature of the PEG polymer, which serves to mitigate the degradation and quenching observed with ICG $[41,45]$. This strategy has also been applied to other NIR dyes, which have subsequently displayed improved solubility, as well as much higher selectivity for drainage through lymphatic vessels following intradermal administration [41]. In particular, it was found that a $20 \mathrm{kDa}$ PEG chain resulted in excellent selectivity for lymphatic drainage while remaining small enough to easily diffuse through the skin [41]. This behavior is well characterized, and such conjugates have been used for NIR lymphatic imaging in a variety of studies since their development [46-48]. However, these conjugates were still delivered through standard intradermal injection, necessitating trained personnel, generating sharp waste, and requiring that analysis be performed in a hospital setting; all of which are obstacles to the development of a home-based lymphedema monitoring system.

Taking advantage on the minimally invasive nature of MNs, and the improved solubility and lymphatic selectivity of PEGylated NIR tracers, we investigated the design of a dissolving polymeric MN patch loaded with a PEG (20 kDa) conjugate of Cyanine 7.5 (Cy7.5), a NIR fluorescent probe. This MN patch was shown to be capable of delivering the PEGylated Cy7.5 tracer to the skin, creating a potential fluorescent medical MN tattoo for lymphedema monitoring. Through rational design, it was found that lymphatic drainage function could be reliably followed using in vivo mouse and rat models. Specifically, 
it was determined that the length of the MNs, as well as the spatial location of the fluorescent tracer within the tips, is critical to limit the high levels of background signal occurring when the dye is not drained from the application site - an issue of great importance for the actual diagnostic potential of the procedure. Additionally, it was shown that this system could be paired with a portable detection technology, demonstrating the potential for simple, minimally-invasive, home-based monitoring of lymphatic drainage [49]. Importantly, we highlight the significance of MN properties, which have important implications for the transdermal delivery of other macromolecules through dissolving polymeric MN systems.

\section{Results and Discussion}

\subsection{Synthesis and Characterization of Cy7.5-PEG}

In this study, Cyanine-7.5 (Cy7.5) was selected as the NIR dye candidate for quantifying lymphatic drainage, as an alternative to the previously-studied ICG. Cy7.5 was chosen based on the availability of a $\mathrm{N}$-hydroxysuccinimide (NHS) ester of the dye, allowing for simple chemical functionalization, which was found to be difficult to accomplish with ICG. Displaying comparable excitation and emission wavelengths to ICG $\left(\lambda_{\mathrm{ex}}=788 \mathrm{~nm}, \lambda_{\mathrm{em}}=808 \mathrm{~nm}\right.$ for Cy7.5; $\lambda_{\mathrm{ex}}=788 \mathrm{~nm}, \lambda_{\mathrm{em}}=$ $813 \mathrm{~nm}$ for ICG) this dye is similarly well-suited for in vivo imaging in terms of spectral properties. However, unconjugated Cy7.5 exhibits poor water solubility (potentially leading to aggregation and cellular uptake) [36,37], and as a small molecule, is susceptible to the same non-specific drainage observed with ICG after intradermal administration [28,49]. To address both of these issues, an activated NHS ester of Cy7.5 was conjugated through amine coupling to a $20 \mathrm{kDa}$ PEG polymer, as this length of PEG was previously found to be optimal for selective lymphatic drainage (Figure 1A) [41]. As expected, the resulting conjugate (Cy7.5-PEG) displayed greatly increased solubility relative to free Cy7.5 (2 mM vs. $3 \mu \mathrm{M}$ ), and is known to be too large and hydrophilic to be taken up by dermal cells (Figure S1). Further, previous studies have shown that $20 \mathrm{kDa}$ PEGylated dyes are large enough to be selectively drained through lymphatic vessels while remaining small enough to diffuse away from the delivery site in the 
dermis when administered through intradermal injection [41-43]. The excitation and emission spectra of Cy7.5-PEG were also examined relative to the unconjugated Cy7.5-NHS ester and no difference was observed, highlighting that the fluorescence properties of the dye are retained after PEGylation (Figure 1B).
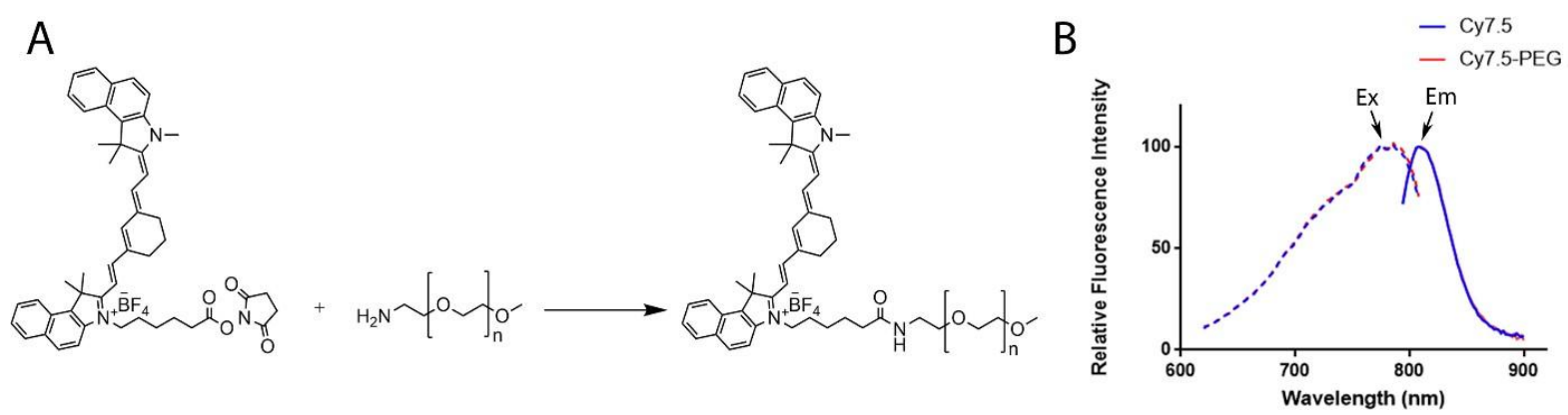

Figure 1. Synthesis and characterization of Cy7.5-PEG: A) Synthesis of Cy7.5-PEG; B) Excitation and emission spectra of Cy7.5 and Cy7.5-PEG.

\subsection{Preparation and Characterization of Dissolving Polymeric MNs}

While earlier studies utilized MNs composed of pure poly( $N$-vinylpyrrolidone) ( $\mathrm{Mw}=3,500-$ 7,000 Da) (PVP K-12), this polymer was found to require long drying times ( $>3$ days) and was associated with fluorophore degradation [28]. Further, it was observed that MNs prepared from PVP K-12 alone were very hygroscopic and required delicate storage conditions to preserve their structure. To address this, MNs were prepared from a 1:1 mixture of PVP K-12 and poly(vinyl alcohol) $(\mathrm{Mw}=10,000$ Da) (PVA) — both injectable polymers commonly used for dissolving MNs — using a solvent casting method in polydimethylsiloxane (PDMS) molds [50-53]. The resulting MNs were found to dry within 24 - 48 hours under ambient conditions and produced sharp, uniform MNs, highlighting the suitability of the polymer blend. In addition to their physical properties, the biocompatibility of both polymers has been widely studied, demonstrating that both PVP and PVA, with molecular weights greater than or equal to those used in our study, undergo rapid renal excretion after injection and have not been found to bioaccumulate [54-56]. Under bright-field microscopy, the MNs appeared as a $15 \times 15$ array of square 
pyramidal projections with average lengths of $479 \pm 13 \mu \mathrm{m}$ and widths of $248 \pm 6 \mu \mathrm{m} \times 248 \pm 6 \mu \mathrm{m}$ at the base (Figure 2A). While the base dimensions of the MNs were in agreement with those of the PDMS molds $(250 \mu \mathrm{m} \times 250 \mu \mathrm{m})$, the lengths were significantly shorter than the nominal lengths of the molds $(600 \mu \mathrm{m})$ (Figure S2), likely due to water loss during the drying process, as previously described [57]. The classical solvent casting process allowed a dye to be dispersed throughout the solid structure of the MNs, including the tips and the backing layer. However, when using MNs for dermal delivery, only the tips dissolve and release their payload, meaning that any dye in the backing layer remains unused. More significantly, dye at the base of the MN tips would primarily be delivered to the stratum corneum and would thus not be drained from the skin, resulting in a high background signal and reducing the sensitivity of the system. This is consistent with observations from previous studies using dissolving polymeric MNs, where strong fluorescence was localized in the outermost regions of the skin after delivery of a fluorescent dye $[33,34,58]$. To resolve this limitation, the solvent casting method was modified to allow dye to be incorporated only in the tips of the MNs, with the backing layer composed of dye-free polymer, by adapting a previously described method [17]. Under fluorescence stereomicroscopy, Cy7.5-PEG fluorescence could be observed exclusively in the tips of the MNs, with no apparent diffusion into the backing layer (Figure 2B). The dye-containing MNs were also examined using scanning electron microscopy (SEM), which revealed smooth, sharp MNs with tip diameters below $10 \mu \mathrm{m}$, sufficient to pierce the skin based on previously reported data (Figure 2C) $[33,59,60]$. 

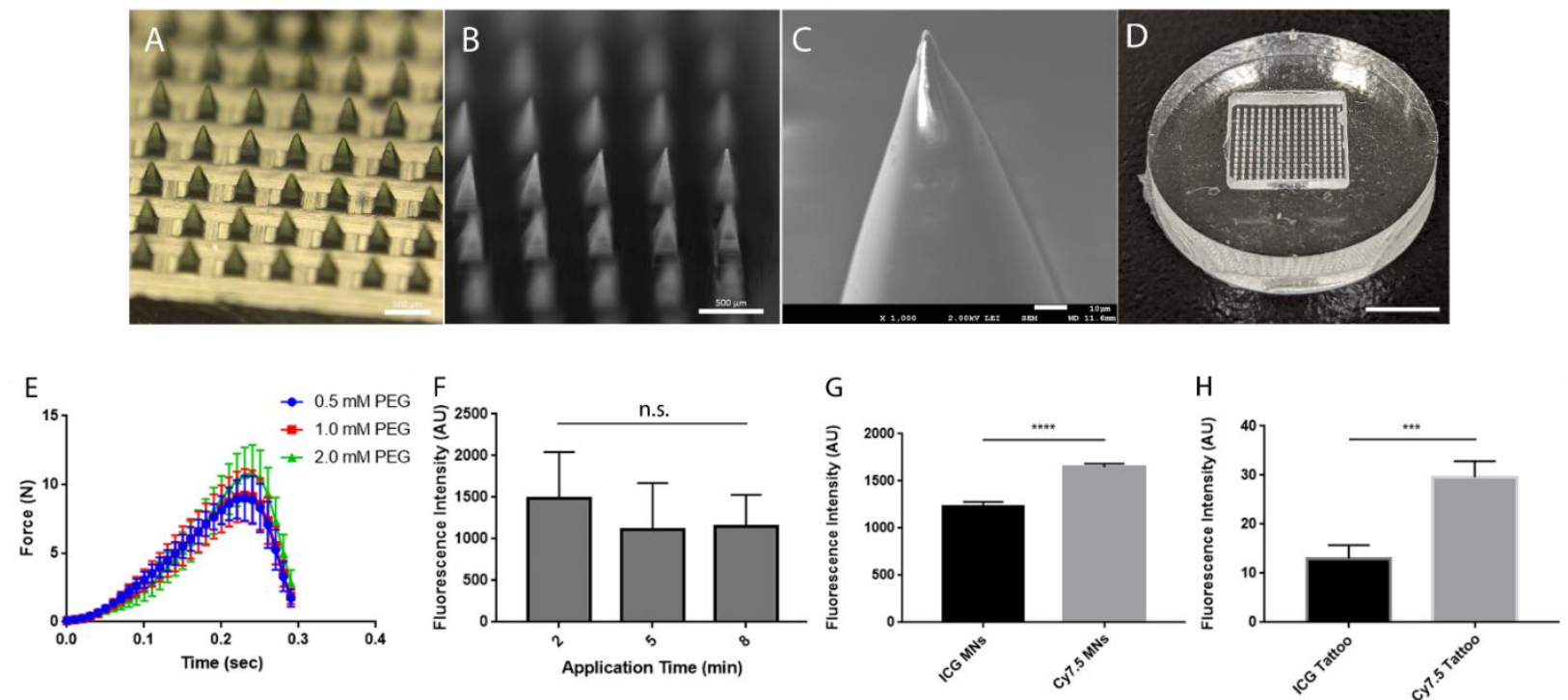

Figure 2. Structure and properties of Cy7.5-PEG-containing polymeric MNs: A) Brightfield image of PVP/PVA (1:1) MNs with Cy7.5-PEG in the tips. Scale bar $=500 \mu \mathrm{m}$; B) Fluorescent image of Cy7.5-PEG localized within MN tips. Scale bar $=500 \mu \mathrm{m}$; C) SEM image of PVP/PVA MNs. Scale bar $=10 \mu \mathrm{m}$; D) Brightfield image of PDMS mold used to make MNs. Scale bar $=5 \mathrm{~mm}$; E) Mechanical properties of PVP/PVA (1:1) MNs with increasing concentrations of PEG-NH $\mathrm{NH}_{2}(20 \mathrm{kDa})$, Mean $\pm \mathrm{SD}(\mathrm{n}$ =4); F) Effect of application time on delivery of Cy7.5-PEG to mouse skin ex vivo, Mean \pm SD ( $=3$ );

G) Fluorescence intensity of MNs containing ICG or Cy7.5-PEG, Mean \pm SD $(n=8), p<0.0001 ; H)$ Fluorescence intensity of ICG or Cy7.5-PEG MN tattoos, Mean \pm SD $(n=4), p \leq 0.0005$.

To ensure that the strength of the MNs was sufficient to pierce the skin, even when large amounts of PEG were loaded, increasing concentrations of PEG-NH $2(20 \mathrm{kDa})$ were incorporated into the MNs and the compression force required to induce structural failure was measured. Ultimately, no significant relationship was observed between failure force and the concentration of $\mathrm{PEG}-\mathrm{NH}_{2}(20 \mathrm{kDa})$, indicating that the concentrations of PEGylated dye used in our experiments did not negatively impact the mechanical properties of the MNs (Figure 2D). Collectively, the tested MNs displayed an average failure force of $9.6 \pm 1.8 \mathrm{~N}$, well above the force required to penetrate human skin (between $0.1 \mathrm{~N}$ and $3 \mathrm{~N}$ ), indicating their suitability for use in vivo [61]. The MNs were examined under brightfield microscopy, 
where it was found that the most common mode of failure was $90^{\circ}$ deflection without breakage of the MN tip (Figure S3).

After confirming that the PVP/PVA MNs possessed the required mechanical strength to breach the skin, their ability to release PEGylated dye within the skin was investigated, as rapid dissolution of the MNs in the skin is a desirable property for eventual clinical translations. Repeated applications of Cy7.5PEG MNs to mouse skin were performed to determine optimal application time; the minimum time required for release of the dye from the MN tips. After testing applications of 2,5, and 8 minutes, it was found that there was no significant difference in the amount of dye delivered, suggesting that a 2 minute application was sufficient for full dissolution of the MN tips and delivery of the maximum possible amount of dye (Figure 2E).

To validate the use of Cy7.5-PEG as an alternative to ICG, MNs were prepared with equivalent concentrations $(720 \mu \mathrm{M})$ of either ICG or Cy7.5-PEG in the tips. After drying, the MN arrays were examined under fluorescence stereomicroscopy and the fluorescence intensities of individual MNs from each array were compared, with Cy7.5-PEG MNs displaying an average increase in fluorescence of $33 \pm 5$ $\%$ relative to their ICG-containing counterparts (Figure 2F). This likely resulted from the partial degradation of ICG in the presence of PVP, as this polymer is known to contain reactive peroxide impurities [49]. In contrast, when MNs containing Cy7.5-PEG were stored for 30 days under ambient conditions, $87 \pm 7 \%$ of fluorescence was retained, indicating the stability of this formulation (Figure S4). MNs of each type were also applied to mouse skin, to compare the intensities of the resulting tattoos (Figure S5). In the skin, the average fluorescence intensity of a Cy7.5-PEG tattoo was found to be $126 \pm$ $41 \%$ greater than the average signal of an ICG tattoo (Figure 2G). This was notable, as, when observed in aqueous solution, the fluorescence intensity of ICG was $22 \pm 17 \%$ greater than that of Cy7.5-PEG at the same concentration (Figure S6). The observed difference in fluorescence intensities could result from aggregation or quenching of ICG within the MNs and the skin, whereas Cy7.5-PEG appeared to exhibit greater solubility and stability in both environments [36,37]. For these reasons, in addition to the 
improved lymphatic selectivity, Cy7.5-PEG was selected for subsequent in vivo studies.

In addition to the stability of the fluorophore, the stability of the Cy7.5-PEG conjugate to hydrolysis was also investigated, to ensure that the dye remained PEGylated once delivered to the dermis. While the amide bond between PEG and the fluorophore is generally recognized as being stable in biological conditions, this had not previously been demonstrated for this specific conjugate [62,63]. To test this, Cy7.5-PEG was incubated in diluted fetal bovine serum (FBS) to mimic conditions found in the ISF of the dermis $[64,65]$, and periodically monitored by LC-MS for the appearance of hydrolyzed free Cy7.5. Ultimately, it was found that the conjugate was fully stable for the first 6 hours of incubation, when drainage was expected to occur, with no hydrolysis observed during this time, indicating sufficient stability for subsequent in vivo studies.

\subsection{In vivo experiments in mice}

Following ex vivo optimization of dye delivery, tests were performed in vivo to determine whether MN-delivered Cy7.5-PEG could successfully be used to monitor lymphatic drainage in mice. To this end, MNs were applied to the shaved skin of mice for 3 minutes, and the resulting fluorescence signal was followed using an IVIS imaging system and a recently developed portable NIR fluorescence detector [49]. The signal was measured periodically over $24 \mathrm{~h}$ (Figure 3A), with a clear decrease in signal observed, following first-order decay kinetics, indicating that the dye was being drained from the application site. The signal initially displayed a steep decline, gradually leveling off into a plateau between $\mathrm{T}=6 \mathrm{~h}$ and $\mathrm{T}=24 \mathrm{~h}$ at $40 \pm 10 \%$ of the initial signal (IVIS) and $32 \pm 11 \%$ of the initial signal (portable device). Notably, the fluorescence signal also displayed no initial dequenching phase, as had previously been reported with ICG, likely due to the improved solubility of the PEGylated Cy7.5, as well as the tendency of ICG to display increased fluorescence intensity upon protein binding [28,38,41,49].

To better quantify the drainage of Cy7.5-PEG, the relative fluorescence of each MN tattoo was plotted as a function of time and the area under the curve (AUC) was measured for each run (Figure 3B). This allowed comparison of the total amount of dye drained for each application, which could ultimately 
be used to quantitatively differentiate between normal lymphatic drainage, and impaired lymphatic drainage in the context of diseases of the lymphatic system. Comparison of the fluorescence measurements collected by the IVIS and the portable fluorescence detector revealed that there was no significant difference between the drainage measured by either instrument $(\mathrm{p} \leq 0.05)$, validating the use of the portable detector for this application (Figure 3C), which holds important implications for monitoring lymphatic drainage outside of a clinical setting [49].

While the kinetics of Cy7.5-PEG drainage were found to be consistent between tests $( \pm 10 \%)$, the initial fluorescence intensity of the tattoos was found to vary more significantly, with the highest intensity application displaying 3.6-fold greater initial fluorescence than the lowest intensity application (Figures S7, S8). This was likely primarily due to variations in skin topography, particularly as the backs of mice acted as small and uneven surfaces for application. Notably, this supports the hypothesis that, due to the inherently relative nature of its quantification, the drainage behavior of the tattoo is independent of the amount of dye delivered (Figure S8). Finally, when comparing the half-life $\left(t_{1 / 2}\right)$ values (corresponding to the speed of drainage from the application site), a small difference was observed, with the portable device indicating shorter $t_{1 / 2}$ values than the IVIS. However, this could be due to diffusion of the dye outside of the smaller field-of-view of the portable device during early stages of drainage, more rapidly lowering the observed fluorescence (Figure 3D).
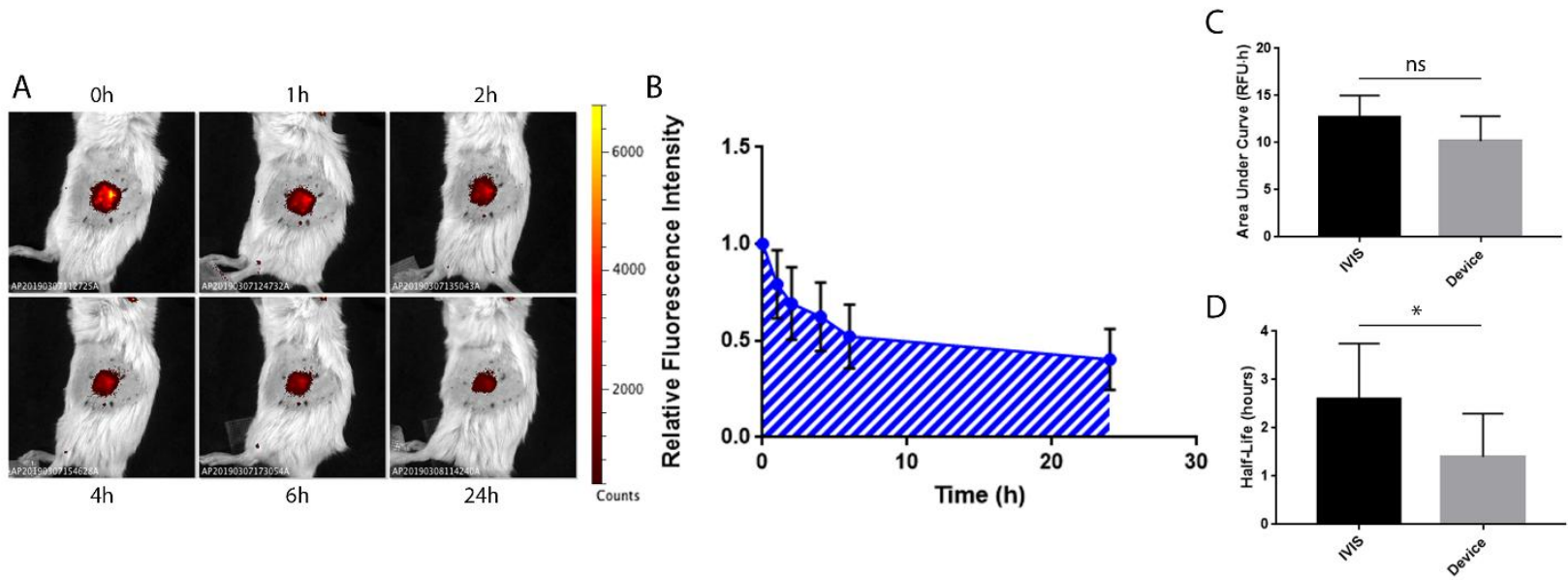

Figure 3. In vivo experiments in mice (IVIS vs portable device): A) Representative IVIS fluorescence 
images of $\mathrm{MN}$ tattoo in mouse at $\mathrm{t}=0,1,2,4,6,24 \mathrm{~h}$; B) Representative AUC plot of relative fluorescence intensity of Cy7.5-PEG in skin, measured using IVIS; C) Comparison between AUCs of measurements performed using IVIS and portable fluorescence detector; D) Comparison between halflife values of Cy7.5-PEG drainage using IVIS and portable fluorescence detector. Mean \pm SD $(n=7)$.

\subsection{In vivo experiments in rats}

Following initial results in mice, further in vivo studies were conducted in rats, a better model for human skin, owing to their similar skin thickness [66]. Specifically, the thickness of rat dorsal (back) skin was reported to be approximately $2 \mathrm{~mm}$, which is comparable to or greater than many regions of the human body, including back, neck, and notably arm skin (a common location of lymphedema following breast cancer-associated axillary lymph node removal) [67]. Cy7.5-PEG-containing MNs of the same geometry and concentration used in mice were applied to the backs of shaved live rats (draining) for 3 minutes, and the resulting fluorescence signal was measured periodically over 24 hours using an IVIS. As rat lymphedema models are complex, requiring microsurgery and/or irradiation to generate [68-70], and primarily affect the extremities - where reproducible $\mathrm{MN}$ application is impossible - MNs were also applied to the backs of shaved dead rats (non-draining) for the same amount of time, to act as negative controls displaying the absence of lymphatic drainage [68,71], to demonstrate the discrimination potential of the MN tattoo. Similar to mice, an initial steep decrease in signal was observed in living rats, with a plateau occurring between $\mathrm{T}=6 \mathrm{~h}$ and $\mathrm{T}=24 \mathrm{~h}$ (Figure 4A). However, this plateau occurred at a significantly higher fluorescence intensity than in mice, with $63 \pm 8 \%$ of the initial signal remaining after 24 hours, suggesting that less Cy7.5-PEG was drained from the application site. This is likely due to the increased thickness of the non-viable epidermis in rats relative to mice, resulting in more dye remaining trapped in this non-drained region of the skin [66]. This was significantly different from the results observed in non-draining rats, where no drainage occurred, and the average $\mathrm{T}=24 \mathrm{~h}$ fluorescence intensity corresponded to $85 \pm 19 \%$ of the initial signal (Figure 4A). The slight initial decrease in fluorescence observed could be attributed to passive diffusion of Cy7.5-PEG within the dermis, away 
from the site of application, or minor changes in the position of the animal upon subsequent measurements. Additionally, the observation that minimal fluorescence is lost in the absence of lymphatic drainage suggests that minimal decay or degradation of the Cy7.5-PEG occurred over the measurement period.

Upon comparison of the AUC values between draining (15.7 $\pm 0.7 \mathrm{RFU} \cdot \mathrm{h})$ and non-draining rats $(21 \pm 2 \mathrm{RFU} \cdot \mathrm{h})$, a significant difference was observed $(\mathrm{p} \leq 0.05)$, suggesting that this $\mathrm{MN}$ tattoo is capable of discrimination between functional and non-functional lymphatic drainage (Figure 4B). The pvalue, corresponding to the statistical difference between the change in fluorescence in the presence or absence of lymphatic drainage, reflects the statistical power of the MN tattoo to discriminate between normal and impaired lymphatic drainage, with lower values indicating an increase in statistical significance. Histological examination of rat skin after MN application revealed significant Cy7.5-PEG fluorescence present in the outer epidermis, with no signal visible deeper in the dermis (Figures 4C, 4D). This further supports the notion that while dye delivered to the dermis or viable epidermis is readily drained through lymphatic vessels, any dye delivered to the outer epidermis remains trapped due to its size and hydrophilicity, resulting in the strong fluorescence after 24 hours $[72,73]$.

A

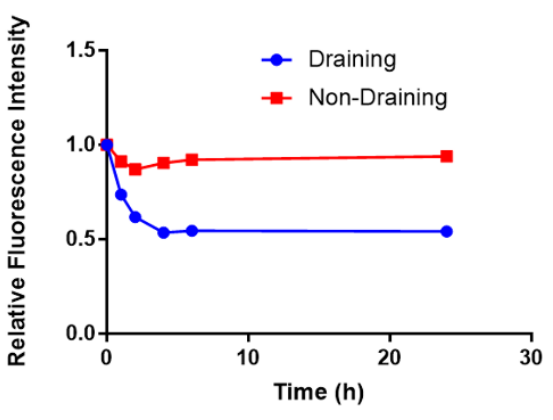

B

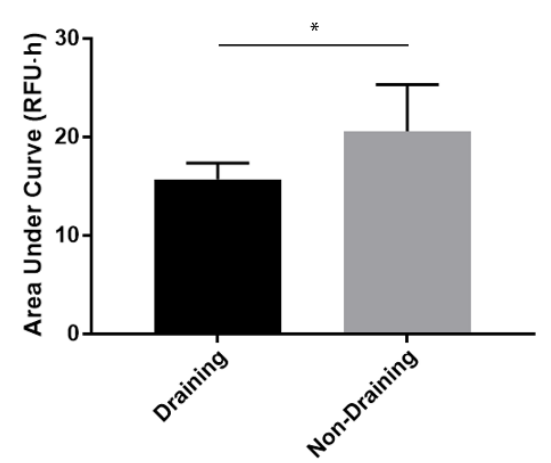

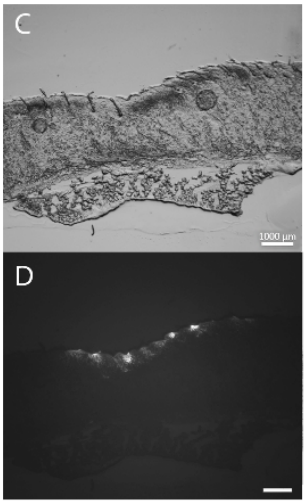

Figure 4. In vivo MN applications in rats: A) Representative relative fluorescence decrease resulting from Cy7.5-PEG drainage in draining and non-draining rats; B) Comparison between AUCs of Cy7.5- 
PEG drainage curves in draining and non-draining rats. Mean \pm SD $(n=6)$; C) Brightfield microscopy image of histology of rat skin after Cy7.5-PEG MNs1 application; D) Fluorescence microscopy image of histology of rat skin showing dye remaining trapped in outer epidermis (MNs were applied while rat was living). Scale bar $=1000 \mu \mathrm{m}$.

\subsection{Optimization of $\mathrm{MN}$ features for in vivo monitoring of lymphatic drainage}

To address the issue of dye remaining trapped in the outer epidermis, and potentially improve the discrimination potential of the system, the MN design was rationally optimized and tested. By modifying the MN preparation process, beginning with the solvent-casting method, the dye was specifically localized within the apex of the tips of the MNs for specific delivery to the deeper, lymphatically drained tissue, allowing greater drainage of the dye and thereby possibly improving the sensitivity of the MN tattoo. Specifically, when prepared using the standard method, the average height of dye within the MNs was measured as $368 \pm 18 \mu \mathrm{m}$, corresponding to $75 \%$ of the total length of the MNs $(479 \pm 13 \mu \mathrm{m})(\mathrm{MNs} 1$, Figure 5A). When prepared using the modified method, Cy7.5-PEG was found to be present up to an average height of $286 \pm 19 \mu \mathrm{m}$, or $60 \%$ of the total MN length (MNs2, Figure 5A). Importantly, to evaluate the impact of delivery depth on the drainage of Cy7.5-PEG, longer MNs were prepared using molds with a nominal height of $800 \mu \mathrm{m}$ (actual MN height measured as $651 \pm 27 \mu \mathrm{m}$ ). When MNs of this length were prepared using the modified casting process, the presence of dye was observed up to a height of $304 \pm 11 \mu \mathrm{m}$, only $45 \%$ of the total MN length (MNs3, Figure 5A). This data was also reflected in ex vivo MN insertion tests in excised skin. Histological examination showed that MNs1 and MNs2 delivered Cy7.5-PEG to average depths of $236 \pm 69 \mu \mathrm{m}$ and $243 \pm 41 \mu \mathrm{m}$ respectively, while the longer MNs3 delivered the dye to a depth of $329 \pm 44 \mu \mathrm{m}$ (Figure 5B, Figure S9). 

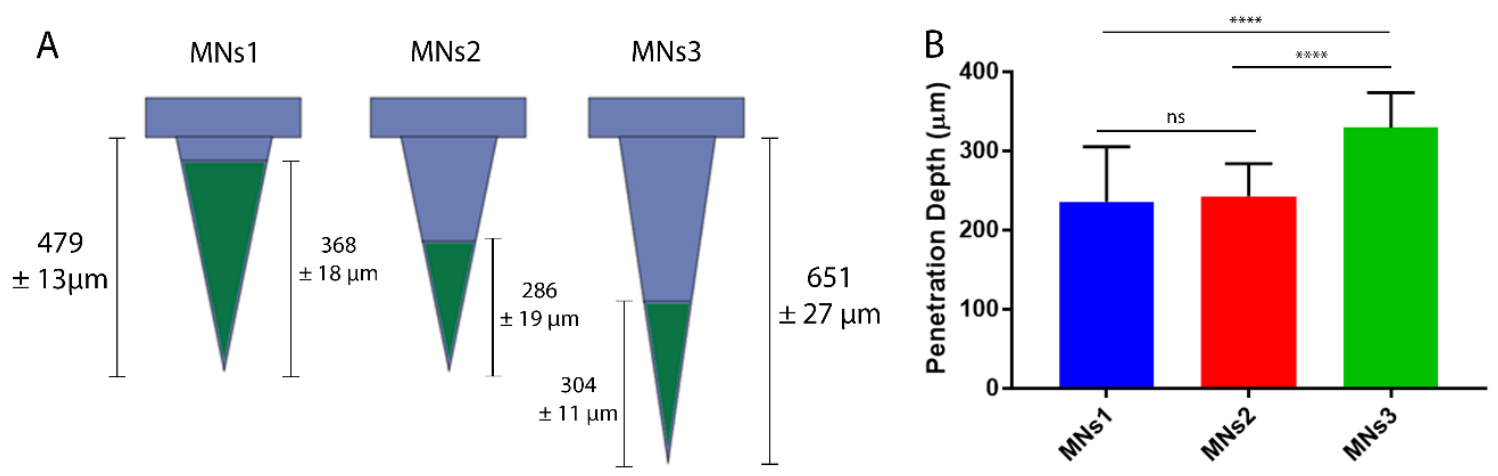

Figure 5: Structure and delivery comparison of MNs1, MNs2, and MNs3: A) Relative heights and location of dye within MNs1, MNs2, and MNs3. Mean \pm SD ( $\mathrm{n}=10)$; B) Average depth of Cy7.5-PEG delivery in porcine skin for MNs1, MNs2 and MNs3. Mean \pm SD $(n=20)$.

These modified MNs were also tested in vivo in draining rats, and the decrease in fluorescence was monitored by IVIS, as previously described. For the MNs2, drainage appeared very similar to previous tests, with an initial decline followed by a slow plateau to $61 \pm 7 \%$ of initial fluorescence. For the MNs3, however, improved drainage was observed, gradually reaching an average $\mathrm{T}=24 \mathrm{~h}$ fluorescence signal of $50 \pm 7 \%$ of the initial signal, lower than previously observed (Figure 6B). Both types of MNs were also applied to non-draining rats, yielding results similar to previous tests $(84 \pm 9 \%$ for MNs2, $91 \pm 22 \%$ for MNs3), with no drainage observed after 24 hours (Figure 6C). Interestingly, the variability of the amount of dye delivered by each class of MNs was similar (Figure S10), further supporting the notion that the rate of drainage does not vary depending on the total amount of dye delivered to the skin.

After application of both MNs2 and MNs3, the AUCs were compared for each MN type, to quantitatively assess their performance. Initially, half-life $\left(t_{1 / 2}\right)$ was also considered for comparison between the different classes of MNs (MNs1, MNs2, MNs3), but it was determined to be poor fit for this. This is because, while $t_{1 / 2}$ is well-suited for comparing rates of lymphatic drainage, it cannot be determined in non-draining rats, as their absence of lymphatic drainage prevents the exponential curvefitting required for half-life determination (Figure S11). The difference between the MN classes is 
instead related to the total amount of dye drained, which is expressed as the area under the curve (AUC). Since the MNs were compared in healthy animals, which should display similar rates of lymphatic drainage, the half-life of the PEGylated conjugate should remain the same, regardless of the class of MNs used to deliver it. However, when comparing the AUCs, a difference was expected, as the different classes of MNs delivered different amounts of dye to the dermis, depending on their length and the location of the dye.

For MNs2, AUCs were very similar to those previously observed, with $15.6 \pm 0.6 \mathrm{RFU} \cdot \mathrm{h}$ for draining rats and $21.0 \pm 0.9 \mathrm{RFU} \cdot \mathrm{h}$ for non-draining rats, though the difference was found to be more significant $(\mathrm{p} \leq 0.0005)$ due to less signal variability between applications (Figure 6D). For the MNs3, the difference was more pronounced, with the average AUC in draining rats measured as $13.7 \pm 0.4 \mathrm{RFU} \cdot \mathrm{h}$, compared to $23 \pm 2 \mathrm{RFU} \cdot \mathrm{h}$ in non-draining rats (Figure 6F). The significance of this difference $(\mathrm{p}<$ 0.0001) was greater than any previously observed, and - coupled with the overall greater drainage observed - suggests that the length of the MNs has an impact on the discrimination potential of the tattoo, likely owing to the presence of less dye in the outer epidermis (Table 1). This was further supported by comparison between the average AUCs of each class of MNs. It was found that the average AUC in draining rats for MNs1 $(15.7 \pm 0.7 \mathrm{RFU} \cdot \mathrm{h})$ was significantly different $(\mathrm{p} \leq 0.05)$ from MNs3 $(13.7 \pm 0.4 \mathrm{RFU} \cdot \mathrm{h})$, but not from MNs2 $(15.6 \pm 0.6 \mathrm{RFU} \cdot \mathrm{h})$, highlighting the improved lymphatic drainage afforded by the optimized MN design.

Additionally, the different classes of MNs were directly compared by considering the relative increase in drainage provided by each class of MNs in draining vs. non-draining rats. This involved normalizing the average AUC from each class of MNs against the mean AUC in non-draining rats to obtain the fold-increase in drainage provided by each class of MNs. In doing so, MNs1 were determined to provide a $1.39 \pm 0.14$-fold increase in draining relative to non-draining animals, while MNs2 and MNs3 provided $1.40 \pm 0.12$-fold and 1.59 \pm 0.16-fold increases respectively (Table 1). This constituted a significant difference $(\mathrm{p} \leq 0.05)$ between MNs1 and MNs3, indicating that the optimized design of MNs3 
provided the best resolution of drainage vs. absence of drainage among the MN designs studied.
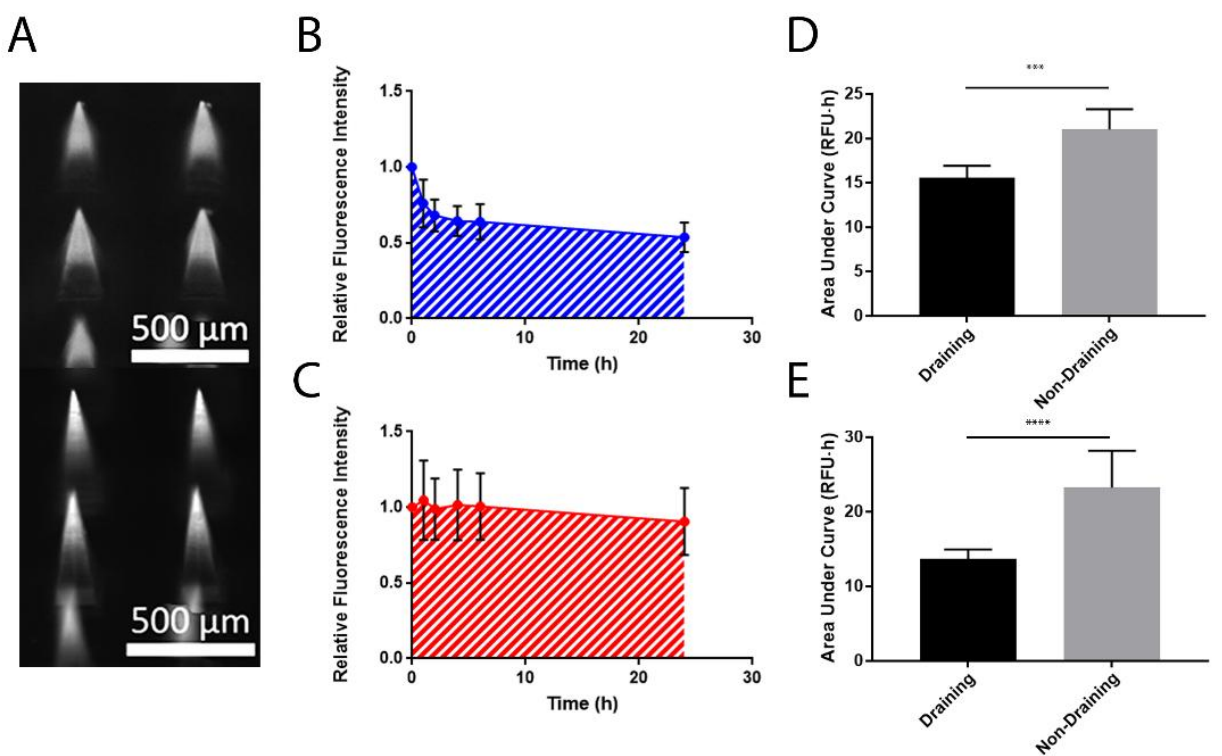

Figure 6. Optimization of MNs for in vivo tests: A) Fluorescence microscopy images of (top to bottom) MNs2, and MNs3; B) AUC plot of relative fluorescence decrease in draining rats of Cy7.5-PEG delivered using MNs3. Mean \pm SD $(n=9)$; C) AUC plot of fluorescence in non-draining rats of Cy7.5-PEG delivered using MNs3. Mean \pm SD $(n=6)$; D) Comparison between AUCs of Cy7.5-PEG drainage curves in draining and non-draining rats using MNs2. Mean $\pm \mathrm{SD}(\mathrm{n}=6)$; E) Comparison between AUCs of Cy7.5-PEG drainage curves in draining and non-draining rats using MNs3. Mean $\pm \operatorname{SD}(n=6)$.

Table 1. Overall properties and in vivo drainage data of different classes of MNs in rats. $\left({ }^{*}\right)$ denotes significance of difference between draining and non-draining rats detectable with each class of MNs; $(*) \mathrm{p} \leq 0.05 ;(* * *) \mathrm{p} \leq$ 0.0005; $(* * * *) \mathrm{p}<0.0001 .(+)$ denotes significance of difference in performance between each class of MNs; $(+) \mathrm{p}$ $\leq 0.05$.

\begin{tabular}{|c|c|c|l|l|l|}
\hline MN & Height of Dye & Delivery & Fluorescence & AUC [RFU·h] & Fold-Increase \\
\hline
\end{tabular}




\begin{tabular}{|l|c|c|c|c|c|c|c|}
\hline & $\begin{array}{c}\text { Length } \\
{[\boldsymbol{\mu m}]}\end{array}$ & Loading $[\boldsymbol{\mu m}]$ & Depth $[\boldsymbol{\mu m}]$ & $\begin{array}{c}\text { Remaining } \\
\text { After 24 h }\end{array}$ & Draining & $\begin{array}{c}\text { Non- } \\
\text { Draining }\end{array}$ & in Drainage \\
\hline MNs1 & $479 \pm 13$ & $368 \pm 18(76.8 \%)$ & $236 \pm 69$ & $63 \pm 8 \%$ & $\begin{array}{c}15.7 \pm 0.7 \\
(*)\end{array}$ & $21 \pm 2$ & $1.39 \pm 0.14$ \\
\hline MNs2 & $479 \pm 13$ & $286 \pm 19(59.8 \%)$ & $243 \pm 41$ & $61 \pm 7 \%$ & $\begin{array}{c}15.6 \pm 0.6 \\
(* * *)\end{array}$ & $21.0 \pm 0.9$ & $1.40 \pm 0.12$ \\
\hline MNs3 & $651 \pm 27$ & $304 \pm 11(46.7 \%)$ & $329 \pm 44$ & $50 \pm 7 \%$ & $13.7 \pm 0.4$ & $23 \pm 2$ & $1.59 \pm 0.16$ \\
\hline
\end{tabular}




\section{Conclusions \& Outlook}

Due to the growing recognition of the importance of the lymphatic vascular system in various diseases, the evaluation and monitoring of its function has rapidly gained interest in the scientific and medical community. In light of this, the system developed in this study represents a step towards a viable MN-based method of lymphatic monitoring. By delivering a PEGylated NIR dye highly selective for lymphatic drainage, we were able to create a $\mathrm{MN}$ tattoo capable of discriminating between lymphatic drainage and the absence of lymphatic drainage in vivo in rats. Through rational optimization of the MN structure, it was determined that the length of the MNs appears to be the most important parameter governing the diagnostic potential of this procedure, owing to the effect on the delivery depth of the dye. In doing so, we identified important parameters for dissolving polymeric MN-based delivery, with great potential significance for future clinical translations of this technology, as well as generally for the delivery of drugs and diagnostic agents with dissolving polymeric MNs. Moreover, it was demonstrated, for the first time, that the lymphatic drainage of a MN tattoo could be measured using a portable fluorescence detector, which constitutes an important clinical advantage for NIR fluorescence imaging of lymphatic drainage, opening the door to at-home and point-of-care monitoring without the need for bulky and expensive detection equipment and trained personnel. Performing this analysis in a minimally invasive manner at home would therefore help improve quality of life for lymphedema patients requiring regular monitoring of lymphatic drainage and allow more rapid detection of changes in health status. Notably, this study also revealed that when delivered using dissolving polymeric MNs, a portion of the PEGylated dye remains in the stratum corneum and is minimally drained — an important consideration for future studies of this class of MNs for drug delivery applications. Finally, while it was demonstrated that the MN tattoo could readily discriminate between drainage vs absence of drainage, this discrimination potential remains to be assessed in clinical settings. In particular, optimal MN length and dye location would need to be evaluated in humans, in order to identify the most sensitive diagnostic procedure. Such studies would be of particular 
importance, given the clinical presentation of lymphedema in humans, which can involve thickening of the skin $[74,75]$. While studies have shown that this thickening is primarily localized to the lymphatically vascularized dermis, rather than the stratum corneum, where dye could remain trapped, this symptom should nonetheless be taken into consideration [74]. It is worth noting, however, that such skin thickening tends to be observed alongside other symptoms (such as visible swelling) during later stages of lymphedema, past the early-detection stage where the MN tattoo would be most useful [76]. This would reveal whether the discrimination potential is sufficient to detect and quantify lymphedema, as well as other diseases related to impairment of lymphatic drainage.

\section{Experimental}

\subsection{Materials}

Poly( $N$-vinylpyrrolidone) $(3.5-7 \mathrm{kDa}) \quad(\mathrm{PVP} \mathrm{K}-12)$ was kindly provided by BASF (Ludwigshafen, Germany). Poly(vinyl alcohol) (10 kDa) (PVA) and fetal bovine serum (FBS) were purchased from Sigma-Aldrich (St. Louis, MO). Indocyanine green (ICG, IR125, laser grade) and dimethyl sulfoxide (DMSO) were purchased from Acros Organics (Morris Plains, NJ). Cyanine 7.5 NHS ester (Cy7.5-NHS) was purchased from Lumiprobe (Hunt Valley, MD). Methoxy-poly(ethylene glycol) amine (MeO-PEG-NH2) (20 kDa) was purchased from JenKem Technologies (Plano, TX). Sephadex G-15 was purchased from GE Life Sciences (Mississauga, ON). Square pyramidal female MN molds made of room temperature vulcanizing silicone, $10 \times 10$ array, $250 \mu \mathrm{m} \times 250 \mu \mathrm{m} \times 800 \mu \mathrm{m}$ $(\mathrm{W} \times \mathrm{L} \times \mathrm{H})$ and $15 \times 15$ array, $250 \mu \mathrm{m} \times 250 \mu \mathrm{m} \times 600 \mu \mathrm{m}(\mathrm{W} \times \mathrm{L} \times \mathrm{H})$ with peak-to-peak spacing of $500 \mu \mathrm{m}$ were purchased from Micropoint Technologies Pte. Ltd. (Singapore).

\subsection{Synthesis of Cy7.5-PEG}

Cy7.5-PEG was synthesized by adapting a previously described procedure [41]. Briefly, Cy7.5NHS (6.0 mg, $7.2 \mu \mathrm{mol}, 1 \mathrm{eq})$ and MeO-PEG-NH $2(\mathrm{MW}=20,000 \mathrm{~g} / \mathrm{mol})(144 \mathrm{mg}, 7.2 \mu \mathrm{mol}, 1 \mathrm{eq})$ were dissolved in anhydrous DMSO $(2 \mathrm{~mL})$, and the mixture was stirred in the dark for $18-24 \mathrm{~h}$. Following this, the mixture was diluted in milli-Q $\mathrm{H}_{2} \mathrm{O}(40 \mathrm{~mL})$ and lyophilized. The resulting residue was 
dissolved in $180 \mathrm{~mm} \mathrm{NaCl}(1 \mathrm{~mL})$ and purified on G-15 Sephadex. The fractions were combined and lyophilized, yielding Cy7.5-PEG as a light green solid (95 mg, $4.5 \mu \mathrm{mol}, 63 \%$ yield). The purity of the product was confirmed by HPLC-MS analysis (Figure S12).

\subsection{Stability of Cy7.5-PEG}

The stability of the Cy7.5-PEG conjugate to hydrolysis was evaluated in FBS diluted to simulate the protein concentration of ISF [64,65]. Briefly, Cy7.5-PEG was dissolved in FBS diluted to $20.6 \mathrm{~g} / \mathrm{L}$ and stored in the dark for $6 \mathrm{~h}$. Periodically, samples were analyzed by HPLC-MS for appearance of a free Cy7.5 peak, indicating hydrolysis.

\subsection{Preparation of Cy7.5-PEG-loaded MNs}

MNs were manufactured by adapting a previously described solvent casting method (Figure S13) [49]. Briefly, to distilled $\mathrm{H}_{2} \mathrm{O}(2 \mathrm{~mL})$, PVP K-12 (0.800 g) and PVA (0.800 g) were added and mixed thoroughly. The mixture was heated for $30-40$ mins in a $75{ }^{\circ} \mathrm{C}$ oven and centrifuged (Sorvall ST 16R, ThermoFisher Scientific, Waltham, MA) for 5 mins at $4700 \mathrm{~g}$, generating a polymer matrix solution (PMS). For the tips, Cy7.5-PEG was added to the matrix solution, resulting in a final concentration of $720 \mu \mathrm{M}$ (PMS1), while for the backing layer, no dye was added (PMS2). Using a $1 \mathrm{~mL}$ syringe, roughly $100 \mu \mathrm{L}$ of PMS1 was cast into PDMS molds (Micropoint Technologies Pte. Ltd., Singapore) and these molds were secured with tape in 6-well cell culture plates (Sarstedt AG \& Co., Nümbrecht, Germany). The plates were covered, secured with parafilm, and centrifuged for 5 mins at $2300 \mathrm{~g}$. After centrifugation, PMS1 was re-applied, and the plates were rotated $180^{\circ}$ and centrifuged again. This process was repeated a total of four times. After the final centrifugation, any excess PMS1 was removed from the molds using a spatula, and they were placed in a vacuum chamber at 150 mbar for 30 minutes. One hundred $\mu \mathrm{L}$ of PMS2 was added to each mold, and they were allowed to dry for 18 $-24 \mathrm{~h}$ at $25{ }^{\circ} \mathrm{C}$ and $60 \%$ humidity, after which the MNs were removed from the molds. For the modified solvent-casting procedure (Figure 5), after removal of excess PMS1 from the molds, a spatula 
was scraped along the bottom of the molds to further remove PMS1 from the MN tips. This was followed by a fifth and final centrifugation using PMS2 to fill the resulting space, vacuum drying at 150 mbar for 30 minutes, and application of a PMS2 backing layer.

\subsection{Characterization of mechanical properties of MNs}

The failure force of the MNs was evaluated using a TA.XT-Plus Texture Analyzer (Stable Micro Systems, Surrey, UK) in compression mode. MNs were prepared as previously described, without addition of dye, and with or without addition of PEG-NH $2(20 \mathrm{kDa})$. MNs were placed on an aluminum plate and a cylindrical stainless-steel probe $(6 \mathrm{~mm}$ diameter $)$ was moved towards the MNs. The probe moved at $1.2 \mathrm{~mm} \cdot \mathrm{s}^{-1}$ with a maximum travel distance of $300 \mu \mathrm{m}$ (sufficient to induce MN deflection). The MN failure force was measured as the maximum of the force-time curve [77].

\subsection{Application of Cy7.5-PEG MNs in vivo in mice}

\section{Animals}

Mice were kept under specific pathogen-free conditions until imaging. FVB mice were bred inhouse at the EPIC animal facility at ETH Zürich. All mouse experiments were carried out in strict accordance with an animal protocol approved by the Cantonal Veterinary Office, Zürich, Switzerland (protocol number: ZH212/16).

\section{In vivo imaging}

The IVIS Spectrum imaging system and the custom portable device were used simultaneously for the in vivo clearance assay in the back skin of mice [49]. Six 19-week-old male FVB mice were used for the assay. Mice were anesthetized with $2 \%$ isoflurane, and fur was removed from the back skin in an area of approximately $3 \mathrm{~cm}$ in diameter using an electric shaver followed by depilating cream (Nair, Church \& Dwight, Ewing, NJ). MNs were applied under anesthesia using a commercially available spring-loaded applicator generating an impact rate of $2 \mathrm{~m} \cdot \mathrm{s}^{-1}$, an impact force of $1.6 \mathrm{~N}$, and with a spring constant of $1 \mathrm{~N} \cdot \mathrm{mm}^{-1}$ (values provided by the manufacturer, Micropoint Technologies Pte. 
Ltd., Singapore) with prolonged contact for 3 minutes for each application. Animals were imaged in an IVIS Spectrum (Caliper Sciences, Hopkinton, MA) in fluorescence mode using a field of view of $6.6 \mathrm{~cm}$ $\times 6.6 \mathrm{~cm}$ (FOV D) with an exposure time of 1.5 seconds, lens aperture (fstop) of f/16 and pixel binning set to 8 . The excitation lamp was set to high intensity with the $745 \mathrm{~nm}$ excitation filter and the ICG emission filter $(810-875 \mathrm{~nm})$. The average fluorescence intensity in a circular ROI $(3 \mathrm{~cm}$ in diameter) was analyzed using Living Image Software. Simultaneously, measurements were performed with the custom portable NIR fluorescence detector. After tracer injection, the circular area $(3 \mathrm{~cm}$ in diameter) around the application site was marked. The device was moved slightly around the marked injection area until the region of highest intensity was detected. Six measurements were then recorded, and the average of the 3 highest values were used for the data analysis. After subtracting the background readout, the values obtained by each instrument were normalized to the time 0 measurement and plotted against time. Animals recovered normally from anesthesia, and displayed no signs of pain or discomfort following MN application.

\subsection{Application of Cy7.5-PEG MNs in vivo in rats}

\section{Animals}

Female Sprague-Dawley rats (Charles River, Senneville, QC) with weights ranging from 400 $500 \mathrm{~g}$ were fed with Alfalfa-free rodent diet (Envigo RMS Inc., Lachine, QC) for 3 weeks prior to experimentation. Experiments were conducted under an ethical protocol approved by the Animal Care Committee of the Faculty of Pharmacy at the University of Montreal (protocol: 19-002) and the Animal Care Council from the Cégep de Lévis-Lauzon (protocol: 002-19).

\section{In vivo imaging}

Rat dorsal skin was shaved and depilated under $2 \%$ isoflurane-induced anesthesia using an electric razor and depilating cream (Nair, Church \& Dwight, Ewing, NJ) the daybefore experimentation. For studies in dead animals, rats were euthanized under anesthesia by cervical dislocation using cutting pliers. Carcasses were kept away from light between scans and kept at $4{ }^{\circ} \mathrm{C}$ for the overnight incubation. 
For living animals, MNs were applied under anesthesia using a commercially available spring-loaded applicator, with a prolonged contact for 3 minutes for each application. Animals were kept away from direct light between imaging sessions.

Animals were imaged in an IVIS Lumina XR (Caliper Sciences, Hopkinton, MA) in fluorescence mode using a field of view of $12.5 \mathrm{~cm} \times 12.5 \mathrm{~cm}(\mathrm{FOV} \mathrm{D})$ with exposure time set to autocompensated, lens aperture (fstop) of f/16 and pixel binning set to 8 . The excitation lamp was set to high with the $745 \mathrm{~nm}$ excitation filter and the ICG emission filter $(810-875 \mathrm{~nm})$. Quantitative data were obtained by drawing square (with constant area) regions of interest (ROIs) around the MN application site to measure fluorescence efficiencies (in \%) which correspond to the quotient of emitted light divided by the excitation light (both in photons/second). After subtracting the background readout, the values obtained were normalized to the time 0 measurement and plotted against time. Areas under the curve (AUCs) were calculated using GraphPad Prism 7 (San Diego, CA). Animals recovered normally from anesthesia, and displayed no signs of pain or discomfort following MN application.

\subsection{Histological Analysis}

The skin area was harvested and frozen at $-80{ }^{\circ} \mathrm{C}$ in Optimal Cutting Temperature (OCT)sucrose compound. Sections of $50 \mu \mathrm{m}$ were cut, chilled by dry ice, and stored at $-80{ }^{\circ} \mathrm{C}$ until imaging for Cy7.5 signal with a fluorescence stereomicroscope (AxioZoom.V16, Zeiss, Oberkochen, Germany) equipped with NIR settings (Excelitas Technologies X-Cite ${ }^{\circledR}$ Xylis light source; Photometrics ${ }^{\circledR}$ Prime $^{\mathrm{TM}}$ 95B camera) using a Cy7 long pass filter set $\left(\lambda_{\mathrm{ex}}=675 \mathrm{~nm}, \lambda_{\mathrm{em}} \geq 730 \mathrm{~nm}\right)$. Sections were then stained with hematoxylin and eosin, and the same regions were imaged under white light excitation (exposure time: 75 and $100 \mathrm{~ms}$ for mouse and rat skin, respectively).

\subsection{Statistical Analysis}

Mean and standard deviation are reported for all data. Significance was analyzed using the Mann-Whitney non-parametric $t$-test $(p$-value $<0.05)$ for comparisons between two datasets (see Figures 2, 3, 4, and 6), or one-way ANOVA ( $p$-value $<0.05$ ) for comparisons between multiple 
datasets (see Figure 5b and Table 1) with Tukey's HSD post hoc test to correct for multiple comparisons. Bartlett's test was used to confirm homogeneity of variance, and the Shapiro-Wilk normality test was used to confirm normality of distribution. All graphs were plotted using GraphPad Prism 7 (San Diego, CA). Statistical significance denoted using *, where $\left(^{*}\right) \mathrm{p} \leq 0.05 ;(* *) \mathrm{p} \leq 0.005$; $(* * *) \mathrm{p} \leq 0.0005 ;(* * * *) \mathrm{p}<0.0001$.

\section{Acknowledgements}

The authors kindly acknowledge Zineb Benkhachan. S.B., and D.B. gratefully acknowledge financial support from the Faculty of Pharmacy at the Universite de Montréal, Natural Sciences and Engineering Research Council of Canada, the Canada Foundation for Innovation, Skintegrity, and the Canadian Generic Pharmaceutical Association and Biosimilars Canada. 


\section{References}

[1] M.A. Swartz, The physiology of the lymphatic system, Adv. Drug Deliv. Rev. 50 (2001) 3-20. https://doi.org/10.1016/s0169-409x(01)00150-8.

[2] G.J. Randolph, S. Ivanov, B.H. Zinselmeyer, J.P. Scallan, The lymphatic system: integral roles in immunity, Annu. Rev. Immunol. 35 (2017) 31-52. https://doi.org/10.1146/annurev-immunol041015-055354.

[3] A.A. Grada, T.J. Phillips, Lymphedema: pathophysiology and clinical manifestations, J. Am. Acad. Dermatol. 77 (2017) 1009-1020. https://doi.org/10.1016/j.jaad.2017.03.022.

[4] S.G. Rockson, K.K. Rivera, Estimating the population burden of lymphedema, Ann. N. Y. Acad. Sci. 1131 (2008) 147-154. https://doi.org/10.1196/annals.1413.014.

[5] S. Bilancini, M. Lucchi, S. Tucci, P. Eleuteri, Functional lymphatic alterations in patients suffering from lipedema, Angiology 46 (1995) 333-339. https://doi.org/10.1177/000331979504600408.

[6] U.K. Franzeck, P. Haselbach, D. Speiser, A. Bollinger, Microangiopathy of cutaneous blood and lymphatic capillaries in chronic venous insufficiency (CVI), Yale J. Biol. Med. 66 (1993) 37-46.

[7] K. Maruyama, J. Asai, M. Ii, T. Thorne, D.W. Losordo, P.A. D’Amore, Decreased macrophage number and activation lead to reduced lymphatic vessel formation and contribute to impaired diabetic wound healing, Am. J. Pathol. 170 (2007) 1178-1191. https://doi.org/10.2353/ajpath.2007.060018.

[8] A. Rossi, F. Sozio, P. Sestini, E.A. Renzoni, K. Khan, C.P. Denton, D.J. Abraham, E. Weber, Lymphatic and blood vessels in scleroderma skin, a morphometric analysis, Hum. Pathol. 41 (2010) 366-374. https://doi.org/10.1016/j.humpath.2009.08.009.

[9] T.J. Ryan, Microcirculation in psoriasis: blood vessels, lymphatics and tissue fluid, Pharmacol. Ther. 10 (1980) 27-64. https://doi.org/10.1016/0163-7258(80)90008-x.

[10] B. Clark, J. Sitzia, W. Harlow, Incidence and risk of arm oedema following treatment for breast cancer: a three-year follow-up study, QJM 98 (2008) 343-348. https://doi.org/10.1093/qjmed/hci053.

[11] S.C. Hayes, M. Janda, B. Cornish, D. Battistutta, B. Newman, Lymphedema after breast cancer: incidence, risk factors, and effect on upper body function, J. Clin. Oncol. 26 (2008) 3536-3542. https://doi.org/10.1200/JCO.2007.14.4899.

[12] C. Shah, D.W. Arthur, D. Wazer, A. Khan, S. Ridner, F. Vicini, The impact of early detection and intervention of breast cancer-related lymphedema: asystemic review, Cancer Med. 5 (2016) 1154 1162. https://doi.org/10.1002/cam4.691.

[13] A. Szuba, W.S. Shin, H.W. Strauss, S. Rockson, The third circulation: radionucleotide lymphoscintigraphy in the evaluation of lymphedema, J. Nucl. Med. 44 (2003) 43-57.

[14] B. Misselwitz, H. Schmitt-Willich, M. Michaelis, J.J. Oellinger, Interstitial magnetic resonance lymphography using a polymeric $t 1$ contrast agent: initial experience with Gadomer-17, Invest. Radiol. 37 (2002) 146-151. https://doi.org/10.1097/00004424-200203000-00007.

[15] A.A. Mancuso, H.R. Harnsberger, A.S. Muraki, M.H. Stevens, Computed tomography of cervical and retropharyngeal lymph nodes: normal anatomy, variants of normal, and applications in staging head and neck cancer. Part II: pathology, Radiology 148 (1983) 715-723.

https://doi.org/10.1148/radiology.148.3.6878692.

[16] J.C. Rasmussen, I.C. Tan, M.V. Marshall, C.E. Fife, E.M. Sevick-Muraca, Lymphatic imaging in humans with near-infrared fluorescence, Curr. Opin. Biotechnol. 20 (2009) 74-82. https://doi.org/10.1016/j.copbio.2009.01.009.

[17] M. Mihara, H. Hara, J. Araki, K. Kikuchi, M. Narushima, T. Yamamoto, T. Iida, H. Yoshimatsu, N. Murai, K. Mitsui, T. Okitsu, I. Koshima, Indocyanine green (ICG) lymphography is superior to lymphoscintigraphy for diagnostic imaging of early lymphedema of the upper limbs, PLoS One 7 (2012) e38182. https://doi.org/10.1371/journal.pone.0038182.

[18] R. Weissleder, A clearer vision for in vivo imaging, Nat. Biotechnol. 19 (2001) 316-317. 
https://doi.org/10.1038/86684.

[19] J. Frangioni, In vivo near-infrared fluorescence imaging, Curr. Opin. Chem. Biol. 7 (2003) 626634. https://doi.org/10.1016/j.cbpa.2003.08.007.

[20] T. Hoshida, N. Isaka, J. Hagendoorn, E. di Tomaso, Y.L. Chen, B. Pytowski, D. Fukumura, T.P. Padera, R.K. Jain, Imaging steps of lymphatic metastasis reveals that vascular endothelial growth factor-C increases metastasis by increasing delivery of cancer cells to lymph nodes: therapeutic implications, Cancer Res. 66 (2006) 8065-8075. https://doi.org/10.1158/0008-5472.CAN-061392.

[21] K. Polom, D. Murawa, P. Nowaczyk, Y.S. Rho, P. Murawa, Breast cancer sentinel lymph node mapping using near infrared guided indocyanine green and indocyanine green--human serum albumin in comparison with gamma emitting radioactive colloid tracer, Eur. J. Surg. Oncol. 38 (2012) 137-142. https://doi.org/10.1016/j.ejso.2011.11.004.

[22] E.L. Jewell, J.J. Huang, N.R. Abu-Rustum, G.J. Gardner, C.L. Brown, Y. Sonoda, R.R. Barakat, D.A. Levine, M.M. Leitao Jr., Detection of sentinel lymph nodes in minimally invasive surgery using indocyanine green and near-infrared fluorescence imaging for uterine and cervical malignancies, Gynecol. Oncol. 133 (2014) 274-277. https://doi.org/10.1016/j.ygyno.2014.02.028.

[23] N. Unno, K. Inuzuka, M. Suzuki, N. Yamamoto, D. Sagara, M. Nishiyama, H. Konno, Preliminary experience with a novel fluorescence lymphography using indocyanine green in patients with secondary lymphedema, J. Vasc. Surg. 45 (2007) 1016-1021.

https://doi.org/10.1016/j.jvs.2007.01.023.

[24] E.M. Sevick-Muraca, R. Sharma, J.C. Rasmussen, M.V. Marshall, J.A. Wendt, H.Q. Pham, E. Bonefas, J.P. Houston, L. Sampath, K.E. Adams, D.K. Blanchard, R.E. Fisher, S.B. Chiang, R. Elledge, M.E. Mawad, Imaging of lymph flow in breast cancer patients after microdose administration of a near-infrared fluorophore: feasbility study, Radiology 246 (2008) 734-741. https://doi.org/10.1148/radiol.2463070962.

[25] R. Philip, A. Penzkofer, W. Bäumler, R. M. Szeimies, C. Abels, Absorption and fluorescence spectroscopic investigation of indocyanine green, J. Photochem. Photobiol. A Chem. 96 (1996) 137-148. https://doi.org/10.1016/1010-6030(95)04292-X.

[26] Y. Xie, T.R. Bagby, M.S. Cohen, M.L. Forrest, Drug delivery to the lymphatic system: importance in future cancer diagnosis and therapies, Expert Opin. Drug Deliv. 6 (2009) 785-792. https://doi.org/10.1517/17425240903085128.

[27] B. Cote, D. Rao, R.G. Alany, G.S. Kwon, A.W.G. Alani, Lymphatic changes in cancer and drug delivery to the lymphatics in solid tumors, Adv. Drug Deliv. Rev. 144 (2019) 16-34. https://doi.org/10.1016/j.addr.2019/08.009.

[28] D. Brambilla, S.T. Proulx, P. Marschalkova, M. Detmar, J.C. Leroux, Microneedles for the noninvasive structural and functional assessment of dermal lymphatic vessels, Small 12 (2016) 1053-1061. https://doi.org/10.1002/smll.201503093.

[29] S. Kaushik, A.H. Hord, D.D. Denson, D.V. McAllister, S. Smitra, M.G. Allen, M.R. Prausnitz, Lack of pain associated with microfabricated microneedles, Anesth. Analg. 92 (2001) 502-504. https://doi.org/10.1097/00000539-200102000-00041.

[30] H.R. Jeong, H.S. Lee, I.J. Choi, J.H. Park, Considerations in the use of microneedles: pain, convenience, anxiety and safety, J. Drug Target. 25 (2017) 29-40. https://doi.org/10.1080/1061186X.2016.1200589.

[31] S. Babity, M. Roohnikan, D. Brambilla, Advances in the design of transdermal microneedles for diagnostic and monitoring applications, Small 14 (2018) e1803186. https://doi.org/ 10.1002/smll.201803186.

[32] H.L. Quinn, E. Larrañeta, R.F. Donnelly, Dissolving microneedles: safety considerations and future perspectives, Ther. Deliv. 7 (2016) 283-285. https://doi.org/10.4155/tde-2016-0015.

[33] J.W. Lee, J.H. Park, M.R. Prausnitz, Dissolving microneedles for transdermal drug delivery, Biomaterials 29 (2008) 2113-2124. https://doi.org/10.1016/j.biomaterials.2007.12.048.

[34] S.P. Sullivan, N. Murthy, M.R. Prausnitz, Minimally invasive protein delivery with rapidly 
dissolving polymer microneedles, Adv. Mater. 20 (2008) 933-938.

https://doi.org/10.1002/adma.200701205.

[35] G.R. Cherrick, S.W. Stein, C.M. Leevy, C.S. Davidson, Indocyanine green: observations on its pjhysical properties, plasma decay, and hepatic extraction, J. Clin. Invest. 39 (1960) 592-600. https://doi.org/10.1172/JCI104072.

[36] J.F. Zhou, M.P. Chin, S.A. Schafer, Aggregation and degradation of indocyanine green, in Proc. SPIE 2128, Laser Surgery: Advanced Characterization, Therapeutics, and Systems IV (7 September 1994). https://doi.org/10.1117/12.184936.

[37] V. Saxena, M. Sadoqi, J. Shao, Degradation kinetics of indocyanine green in squeous solution, J. Pharm. Sci. 92 (2003) 2090-2097. https://doi.org/10.1002/jps.10470.

[38] M. Weiler, T. Kassis, J.B. Dixon, Sensitivity analysis of near-infrared functional lymphatic imaging, J. Biomed. Opt. 17 (2012) 066019. https://doi.org/10.1117/1.JBO.17.6.066019.

[39] A.A. Gashev, T. Nagai, E.A. Bridenbaugh, Indocyanine Green and lymphatic imaging: current problems, Lymph. Res. Biol. 8 (2010) 127-130. https://doi.org/10.1089/1rb.2010.0005.

[40] M. Weiler, J.B. Dixon, Differential transport function of lymphatic vessels in the rat tail model and the long-term effects of Indocyanine Green as assessed with near-infrared imaging, Front. Physiol. 4 (2013) 215. https://doi.org/10.3389/fphys.2013.00215.

[41] S.T. Proulx, P. Luciani, A. Christiansen, S. Karaman, K.S. Blum, M. Rinderknecht, J.C. Leroux, M. Detmar, Use of a PEG-conjugated bright near-infrared dye for functional imaging of rerouting of tumor lymphatic drainage after sentinel lymph node metastasis, Biomaterials 34 (2013) 51285137. https://doi.org/10.1016/j.biomaterials.2013.03.034.

[42] L.M. Kaminskas, D.B. Ascher, V.M. McLeod, M.J. Herold, C.P. Le, E.K. Sloan, C.J. Porter, PEGylation of interferon $\alpha 2$ improves lymphatic exposure after subcutaneous and intravenous administration and improves antitumour efficacy against lymphatic breast cancer metastases, J. Control. Release 168 (2013) 200-208. https://doi.org/10.1016/j.jconrel.2013.03.006.

[43] N.L. Trevaskis, L.M. Kaminskas, C.J. Porter, From sewer to saviour - targeting the lymphatic system to promote drug exposure and activity, Nat. Rev. Drug Discov. 14 (2015) 781-803. https://doi.org/10.1038/nrd4608.

[44] L.J. Chan, D.B. Ascher, R. Yadav, J.B. Bulitta, C.C. Williams, C.J. Porter, C.B. Landersdorfer, L.M. Kaminskas, Conjugation of $10 \mathrm{kDa}$ Linear PEG onto Trastuzumab Fab' Is Sufficient to Significantly Enhance Lymphatic Exposure while Preserving in Vitro Biological Activity, Mol. Pharm. 13 (2016) 1229-1241. https://doi.org/10.1021/acs.molpharmaceut.5b00749.

[45] J.M. Harris, N.E. Martin, M. Modi, Pegylation: a novel process for modifying pharmacokinetics, Clin. Pharmacokinet. 40 (2001) 539-551. https://doi.org/10.2165/00003088-200140070-00005.

[46] S.T. Proulx, Q. Ma, D. Andina, J.-C. Leroux, M. Detmar, Quantitative measurement of lymphatic function in mice by noninvasive near-infrared imaging of a peripheral vein, JCI Insight 2 (2017) e90861. https://doi.org/10.1172/jci.insight.90861.

[47] Q. Ma, Y. Decker, A. Müller, B.V. Ineichen, S.T. Proulx, Clearance of cerebrospinal fluid from the sacral spine through lymphatic vessels, J. Exp. Med. 216 (2019) 2492-2502. https://doi.org/10.1084/jem.20190351.

[48] S.B. Bachmann, S.T. Proulx, Y. He, M. Ries, M. Detmar, Differential effects of anaesthesia on the contractility of lymphatic vessels in vivo, J. Physiol. 597 (2019) 2841-2852. https://doi.org/10.1113/JP277254.

[49] A.K. Polomska, S.T. Proulx, D. Brambilla, D. Fehr, M. Bonmarin, S. Brändli, M. Meboldt, C. Steuer, T. Vasileva, N. Reinke, J.C. Leroux, M. Detmar, Minimally invasive method for the pointof-care quantification of lymphatic vessel function, JCI Insight 4 (2019) e126515. https://doi.org/ 10.1172/jci.insight.126515.

[50] S. Indermun, R. Luttge, Y.E. Choonara, P. Kumar, L.C. du Toit, G. Modi, V. Pillay, Current advances in the fabrication of microneedles for transdermal delivery, J. Control. Release 185 (2014) 130-138. https://doi.org/10.1016/j.jconrel.2014.04.052.

[51] L.Y. Chu, M.R. Prausnitz, Separable arrowhead microneedles, J. Control. Release 149 (2011) 
242-249. https://doi.org/10.1016/j.jconrel.2010.10.033.

[52] I.C. Lee, J.S. He, M.T. Tsai, K.C. Lin, Fabrication of a novel partial dissolving polymer microneedle patch for transdermal drug delivery, J. Mater. Chem. B 3 (2015) 276-285. https://doi.org/10.1039/C4TB01555J.

[53] M. Wang, L. Hu, C. Xu, Recent advances in the design of polymeric microneedles for transdermal drug delivery and biosensing, Lab Chip 17 (2017) 1373-1387.

https://doi.org/10.1039/C7LC00016B

[54] L. Arisz, B.P. Hazenberg, A. van Zanten, E. Mandema, Renal excretion of low and high molecular weight polyvinylpyrrolidone (PVP) in patients with proteinuria, Acta Med. Scand. 186 (1969) 393-400. https://doi.org/10.1111/j.0954-6820.1969.tb01492.x.

[55] J.M. Sanders, H.B. Matthews, Vaginal absorption of polyvinyl alcohol in Fischer 344 rats, Hum. Exp. Toxicol. 9 (1990) 71-78. https://doi.org/10.1177/096032719000900202.

[56] C.E. Hall, O. Hall, Polyvinyl alcohol nephrosis: relationship of degree of polymerization to pathophysiologic effects, Proc. Soc. Exp. Biol. Med. 112 (1963) 86-91. https://doi.org/10.3181/00379727-112-27958.

[57] S. Yang, Y. Feng, L. Zhang, N. Chen, W. Yuan, T. Jin, A scalable fabrication process of polymer microneedles, Int. J. Nanomedicine 7 (2012) 1415-1422. https://doi.org/10.2147/IJN.S28511.

[58] S.P. Sullivan, D.G. Koutsonanos, M. del Pilar Martin, J.W. Lee, V. Zarnitsyn, S.O. Choi, N. Murthy, R.W. Compans, I. Skountzou, M.R. Prausnitz, Dissolving polymer microneedle patches for influenza vaccination, Nat. Med. 16 (2010) 915-920. https://doi.org/10.1038/nm.2182.

[59] K. Lee, C.Y. Lee, H. Jung, Dissolving microneedles for transdermal drug administration prepared by stepwise controlled drawing of maltose, Biomaterials 32 (2011) 3134-3140. https://doi.org/10.1016/j.biomaterials.2011.01.014.

[60] S.G. Lee, J.H. Jeong, K.M. Lee, K.H. Jeong, H. Yang, M. Kim, H. Jung, S. Lee, Y.W. Choi, Nanostructured lipid carrier-loaded hyaluronic acid microneedles for controlled dermal delivery of a lipophilic molecule, Int. J. Nanomedicine 9 (2014) 289-299. https://doi.org/10.2147/IJN.S54529.

[61] S.P. Davis, B.J. Landis, Z.H. Adams, M.G. Allen, M.R. Prausnitz, Insertion of microneedles into skin: measurement and prediction of insertion force and needle fracture force, J. Biomech. 37 (2004) 1155-1163. https://doi.org/10.1016/j.jbiomech.2003.12.010.

[62] K.L. Amsberry, R.T. Borchardt, Amine prodrugs which utilize hydroxy amide lactonization. I. A potential redox-sensitive amide prodrug, Pharm. Res. 8 (1991) 323-330. https://doi.org/10.1023/A:1015885213625.

[63] V.I. Martynov, A.A. Pakhomov, N.V. Popova, I.E. Deyev, A.G. Petrenko, Synthetic fluorophores for visualizing biomolecules in living systems, Acta Naturae 8 (2016) 33-46. https://doi.org/10.32607/20758251-2016-8-4-33-46.

[64] N. Fogh-Anderson, B.M. Altura, B.T. Altura, O. Siggaard-Andersen, Composition of interstitial fluid, Clin. Chem. 41 (1995) 1522-1525. https://doi.org/10.1093/clinchem/41.10.1522.

[65] I.V. Branzoi, M. Iordoc, F. Branzoi, R. Vasilescu-Mirea, G. Sbarcea, Influence of diamond-like carbon coating on the corrosion resistence of the NITINOL shape memory alloy, Surf. Interface Anal. 42 (2010) 502-509. https://doi.org/10.1002/sia.3473.

[66] N.A. Monteiro-Riviere, D.G. Bristol, T.O. Manning, R.A. Rogers, J.E. Riviere, Interspecies and interregional analysis of the comparative histologic thickness and laser Doppler blood flow measurements at five cutaneous sites in nine species, J. Invest. Dermatol. 95 (1990) 582-586. https://doi.org/10.1111/1523-1747.ep12505567.

[67] Y. Lee, K. Hwang, Skin thickness of Korean adults, Surg. Radiol. Anat. 24 (2002) 183-189. https://doi.org/10.1007/s00276-002-0034-5.

[68] G.Y. Wang, S.Z. Zhong, A model of experimental lymphedema in rats' limbs, Microsurgery 6 (1985) 204-210. https://doi.org/10.1002/micr.1920060404.

[69] M.A. Kanter, S.A. Slavin, W. Kaplan, An experimental model for chronic lymphedema, Plast. Reconstr. Surg. 85 (1990) 573-580. https://doi.org/10.1097/00006534-199004000-00012.

[70] L. Lee-Donaldson, M.H. Witte, M. Bernas, C.L. Witte, D. Way, B. Stea, Refinement of a rodent 
model of peripheral lymphedema, Lymphology 32 (1999) 111-117.

[71] F. Serizawa, K. Ito, M. Matsubara, A. Sato, H. Shimokawa, S. Satomi, Extracorporeal shock wave therapy induces therapeutic lymphangiogenesis in a rat model of secondary lymphoedema, Eur. J. Vasc. Endovasc. Surg. 42 (2011) 254-260. https://doi.org/10.1016/j.ejvs.2011.02.029.

[72] J.D. Bos, M.M. Meinardi, The 500 Dalton rule for the skin penetration of chemical compounds and drugs, Exp. Dermatol. 9 (2000) 165-169. https://doi.org/10.1034/j.16000625.2000.009003165.x.

[73] B.C. Palmer, L.A. DeLouise, Nanoparticle-Enabled Transdermal Drug Delivery Systems for Enhanced Dose Control and Tissue Targeting, Molecules 21 (2016) e1719. https://doi.org/10.3390/molecules21121719.

[74] R.H. Mellor, N.L. Bush, A.W.B. Stanton, J.C. Bamber, J.R. Levick, P.S. Mortimer, Dualfrequency ultrasound examination of skin and subcutis thickness in breast cancer-related lymphedema, Breast J. 10 (2004) 496-503. https://doi.org/ 10.1111/j.1075-122X.2004.21458.x.

[75] S.U. Shin, W. Lee, E.-A. Park, C.-I. Shin, J.W. Chung, J.H. Park, Comparison of characteristic CT findings of lymphedema, cellulitis, and generalized edema in lower leg swelling, Int. J. Cardiovasc. Imaging 29 (2013) 135-143. https://doi.org/10.1007/s10554-013-0332-5.

[76] M. Marotel, R. Cluzan, S. Ghabboun, M. Pascot, F. Alliot, J.L. Lasry, Transaxial computer tomography of lower extremity lymphedema, Lymphology 31 (1998) 180-185.

[77] E. Larrañeta, R.E. Lutton, A.J. Brady, E.M. Vicente-Pérez, A.D. Woolfson, R.R. Thakur, R.F. Donnelly, Microwave-assisted preparation of hydrogel-forming microneedle arrays for transdermal drug delivery applications, Macromol. Mater. Eng. 300 (2015) 586-595. https://doi.org/10.1002/mame.201500016. 


\section{Figures, Schemes, and Legends}

Figure 1. Synthesis and characterization of Cy7.5-PEG.

Figure 2. Structure and properties of Cy7.5-PEG-containing polymeric MNs.

Figure 3. In vivo experiments in mice (IVIS vs portable device).

Figure 4. In vivo MN applications in rats.

Figure 5. The relative heights and location of dye within MNs1, MNs2, and MNs3.

Figure 6. Optimization of MNs for in vivo tests.

\section{Tables}

Table 1. Overall properties and in vivo drainage data of different classes of MNs in rats.

\section{Table of Contents}

The lymphatic system is involved in a variety of diseases, and its monitoring is of great interest in medicine. Existing methods of imaging the lymphatic system often require expensive and complex detection. Microneedles present a novel, minimally invasive method of imaging the lymphatic system, by delivering a specially designed near-infrared probe as a fluorescent medical tattoo, compatible with portable detection.
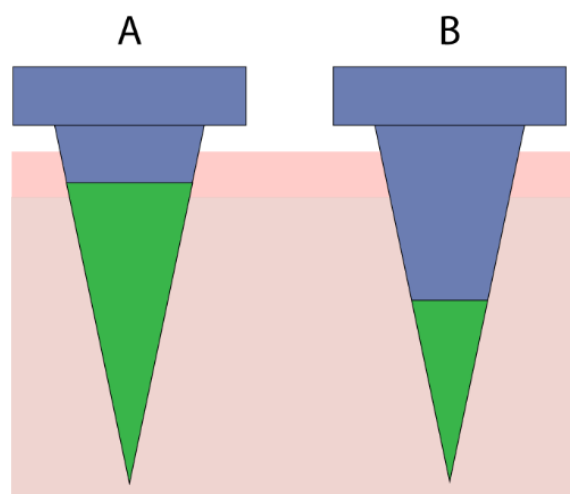

\section{Skin}
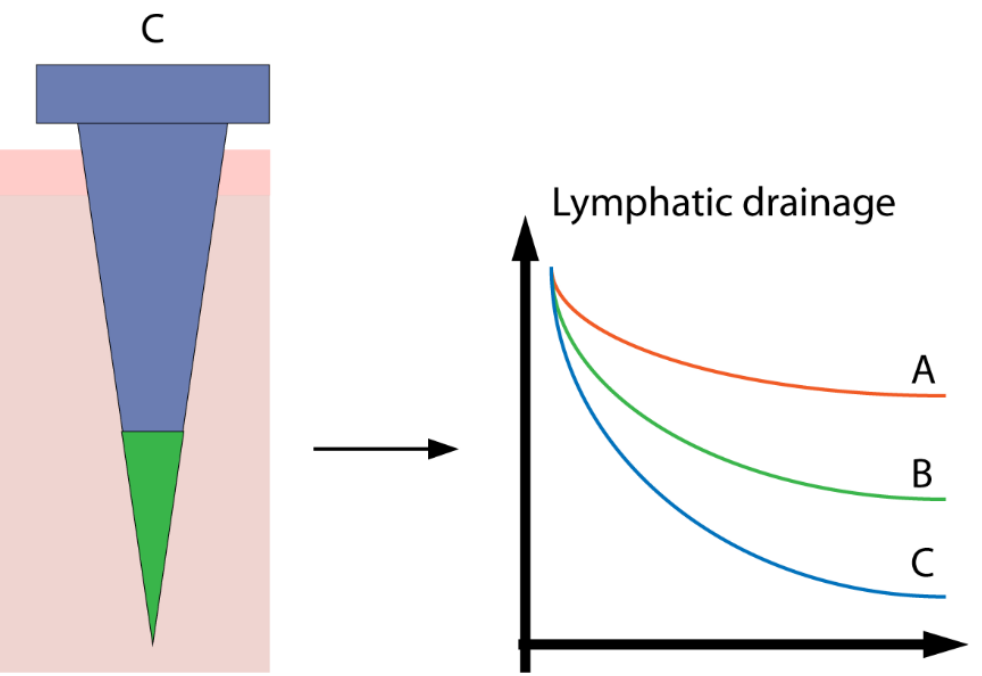

Keyword: Microneedles. 
Table 1. Overall properties and in vivo drainage data of different classes of MNs in rats. (*) denotes significance of difference between draining and non-draining rats detectable with each class of MNs; $(*) \mathrm{p} \leq 0.05 ;\left({ }^{* * *}\right) \mathrm{p} \leq 0.0005 ;\left({ }^{* * * *}\right) \mathrm{p}<0.0001 .(+)$ denotes significance of difference in performance between each class of MNs; $(+) \mathrm{p} \leq 0.05$.

\begin{tabular}{|c|c|c|c|c|c|c|c|}
\hline & \multirow{2}{*}{$\begin{array}{c}\text { MN } \\
\text { Length } \\
{[\mu \mathrm{m}]}\end{array}$} & \multirow{2}{*}{$\begin{array}{c}\text { Height of } \\
\text { Dye Loading } \\
{[\mu \mathrm{m}]}\end{array}$} & \multirow{2}{*}{$\begin{array}{c}\text { Delivery } \\
\text { Depth } \\
{[\mu \mathrm{m}]}\end{array}$} & \multirow{2}{*}{$\begin{array}{c}\text { Fluorescence } \\
\text { Remaining } \\
\text { After } 24 \mathrm{~h}\end{array}$} & \multicolumn{2}{|c|}{ AUC $[\mathbf{R F U} \cdot \mathbf{h}]$} & \multirow{2}{*}{$\begin{array}{c}\text { Fold- } \\
\text { Increase in } \\
\text { Drainage }\end{array}$} \\
\hline & & & & & Draining & $\begin{array}{c}\text { Non- } \\
\text { Draining }\end{array}$ & \\
\hline MNs1 & $479 \pm 13$ & $\begin{array}{l}368 \pm 18 \\
(76.8 \%)\end{array}$ & $236 \pm 69$ & $63 \pm 8 \%$ & $\begin{array}{c}15.7 \pm 0.7 \\
(*)\end{array}$ & $21 \pm 2$ & $1.39 \pm 0.14$ \\
\hline MNs2 & $479 \pm 13$ & $\begin{array}{l}286 \pm 19 \\
(59.8 \%)\end{array}$ & $243 \pm 41$ & $61 \pm 7 \%$ & $\begin{array}{c}15.6 \pm 0.6 \\
(* * *)\end{array}$ & $21.0 \pm 0.9$ & $1.40 \pm 0.12$ \\
\hline MNs3 & $651 \pm 27$ & $\begin{array}{l}304 \pm 11 \\
(46.7 \%)\end{array}$ & $329 \pm 44$ & $50 \pm 7 \%$ & $\begin{array}{l}13.7 \pm 0.4 \\
(* * * *)(+)\end{array}$ & $23 \pm 2$ & $1.59 \pm 0.16$ \\
\hline
\end{tabular}


Click here to download high resolution image

A

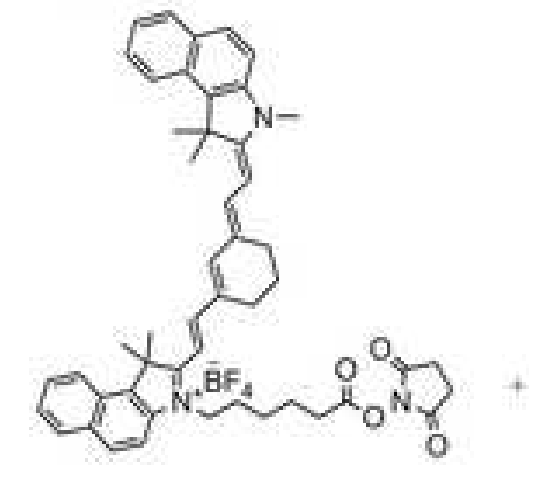

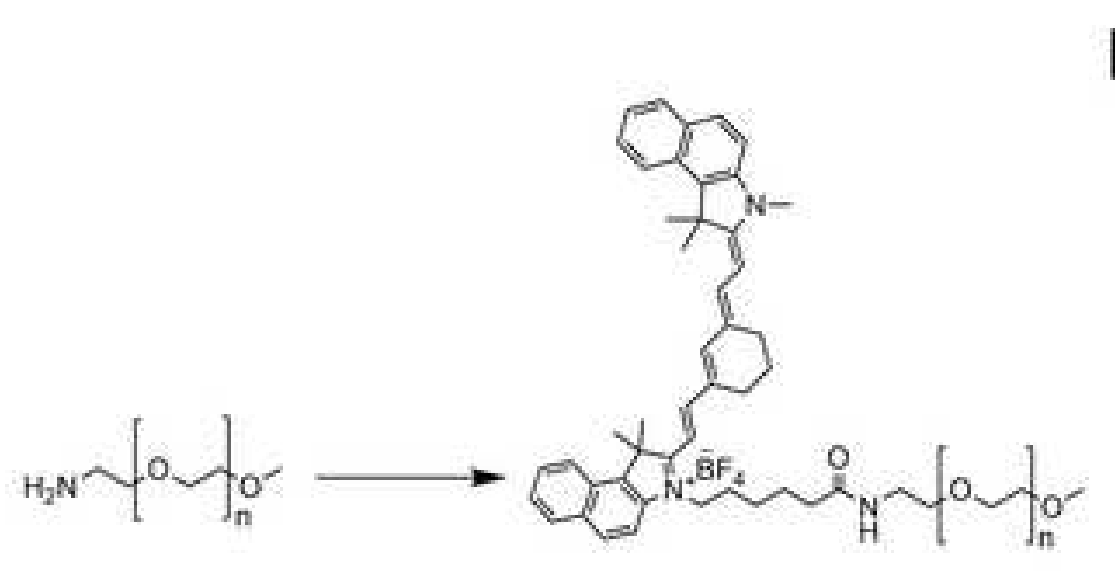

B

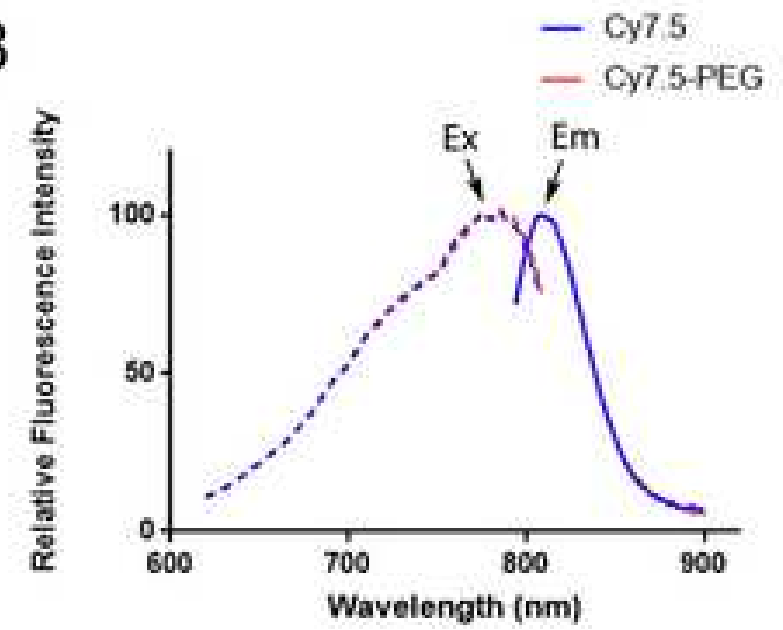


Click here to download high resolution image
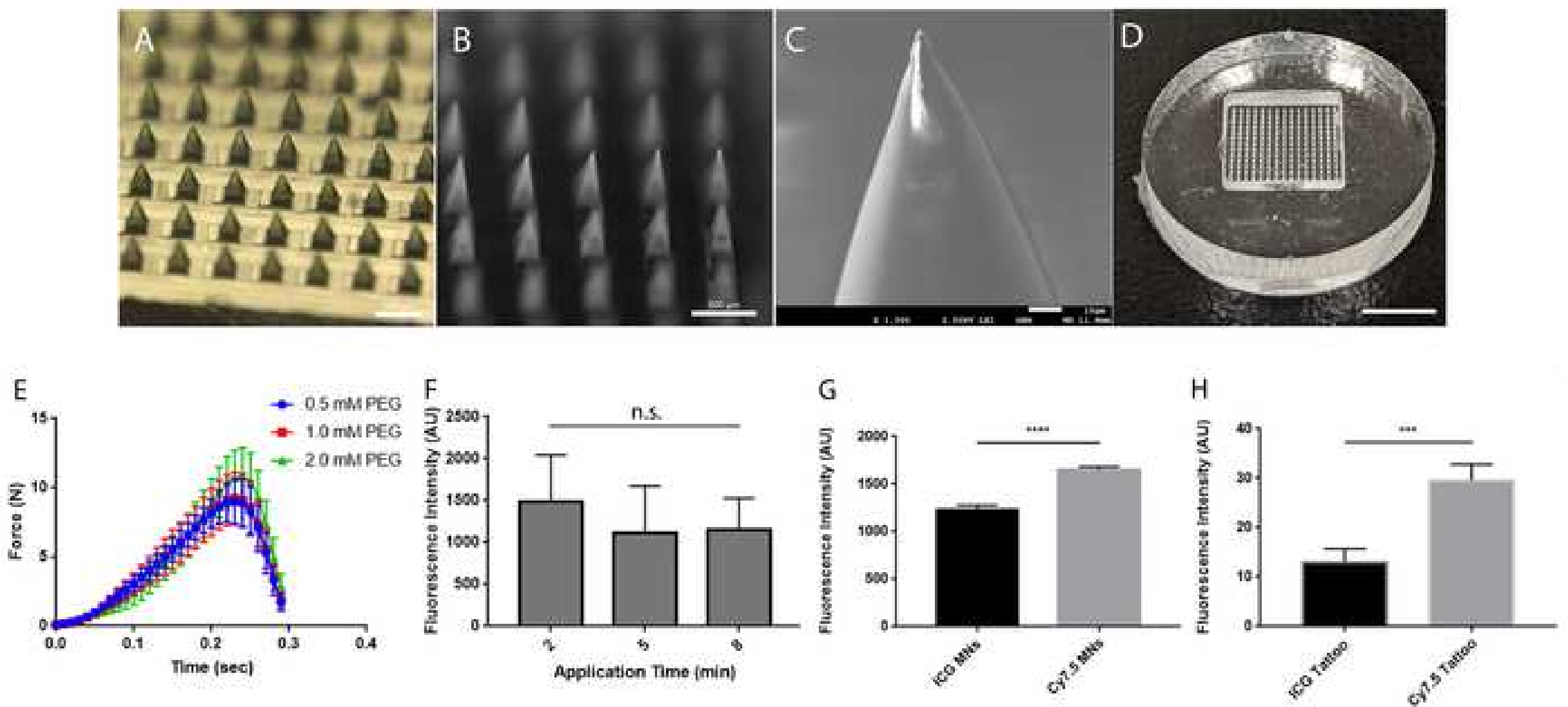


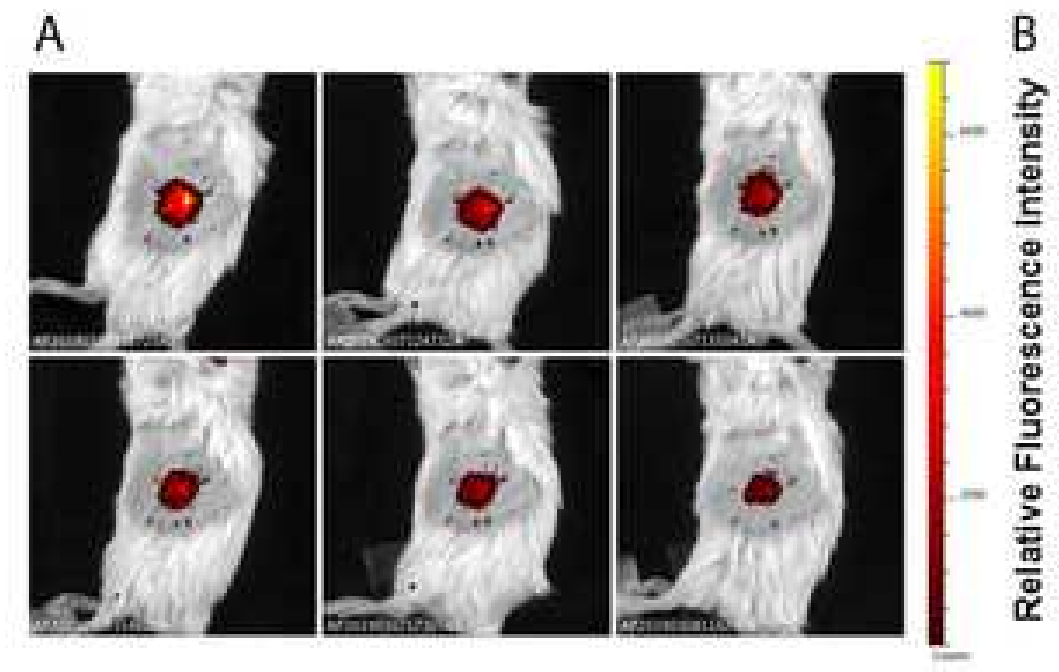

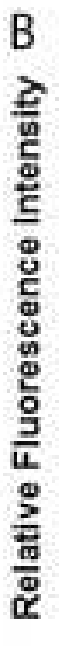

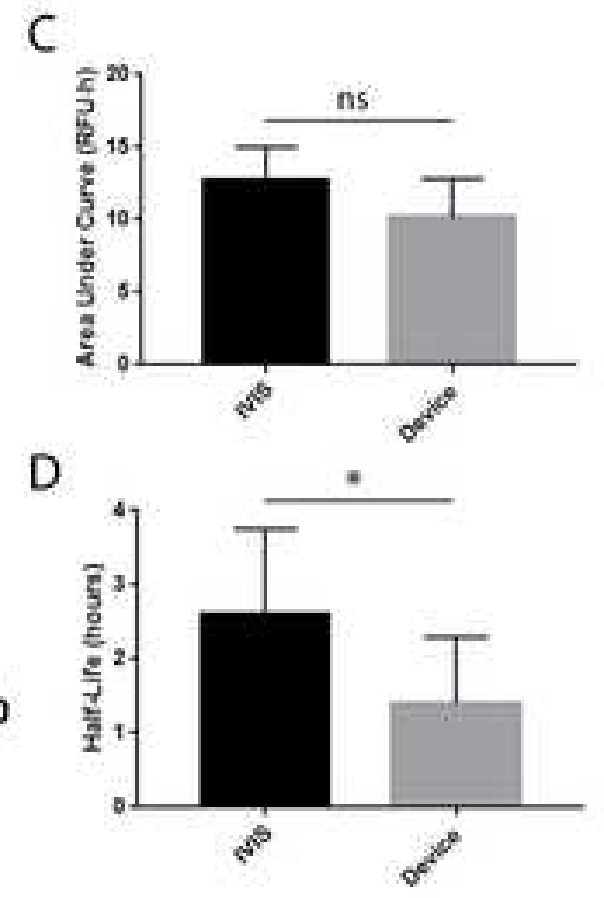


Click here to download high resolution image
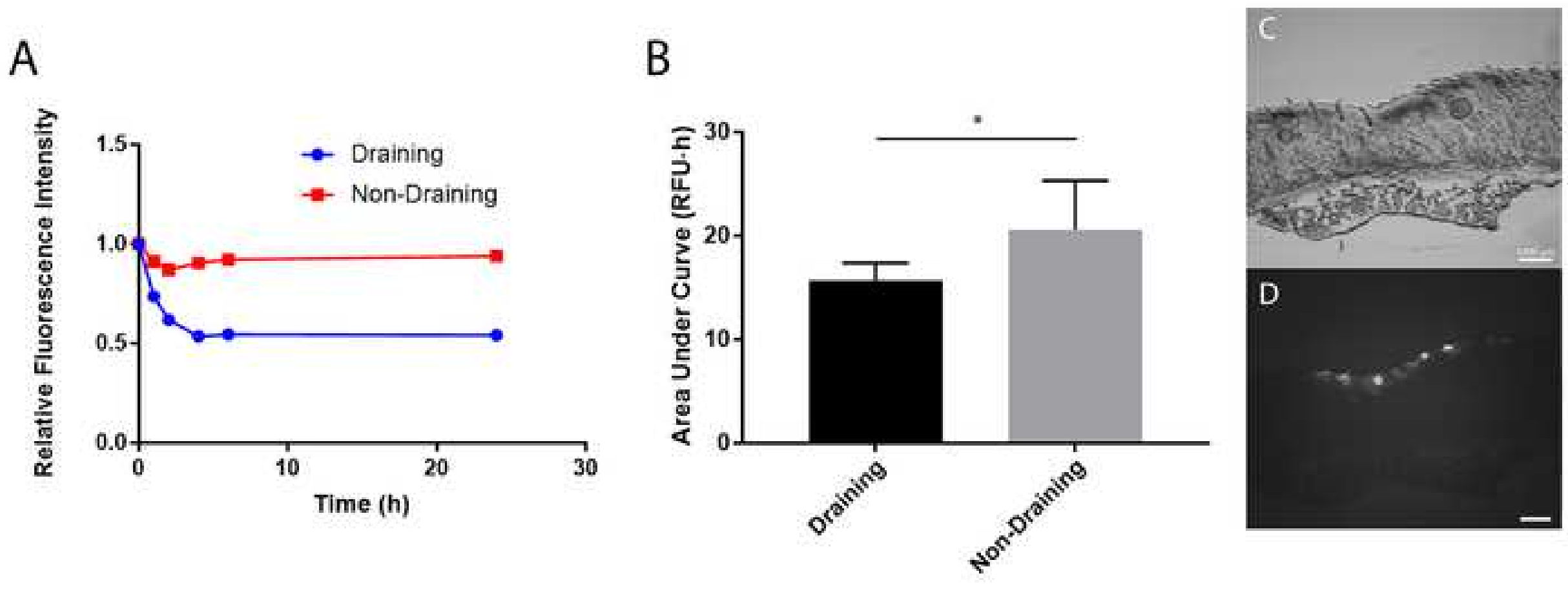
Click here to download high resolution image
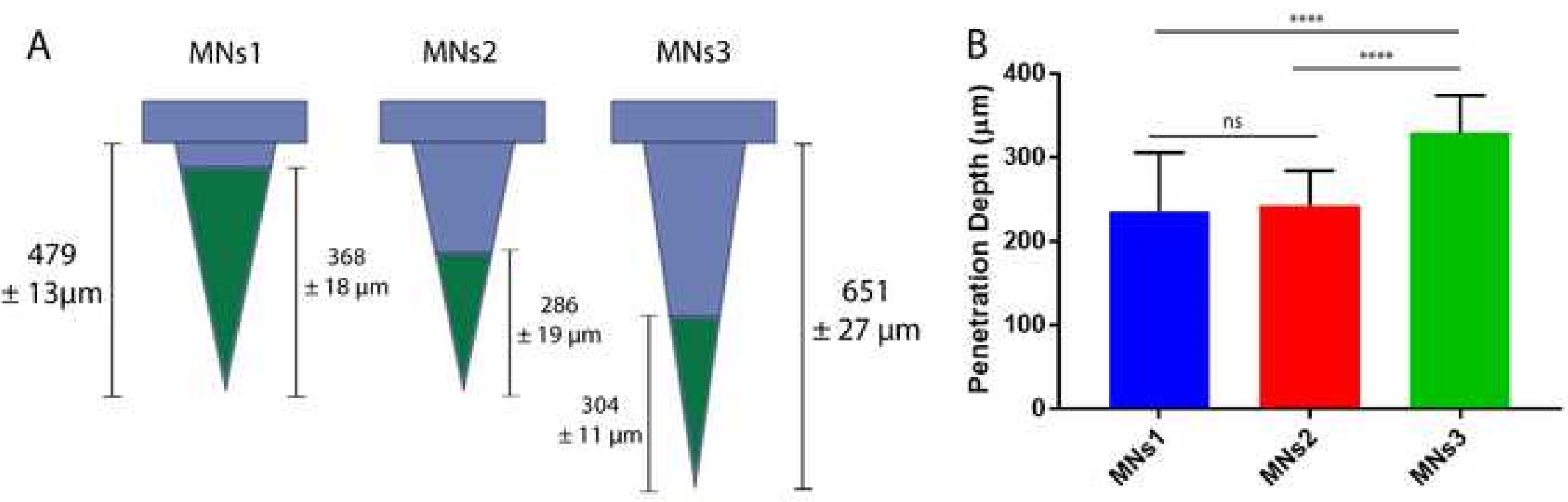
A

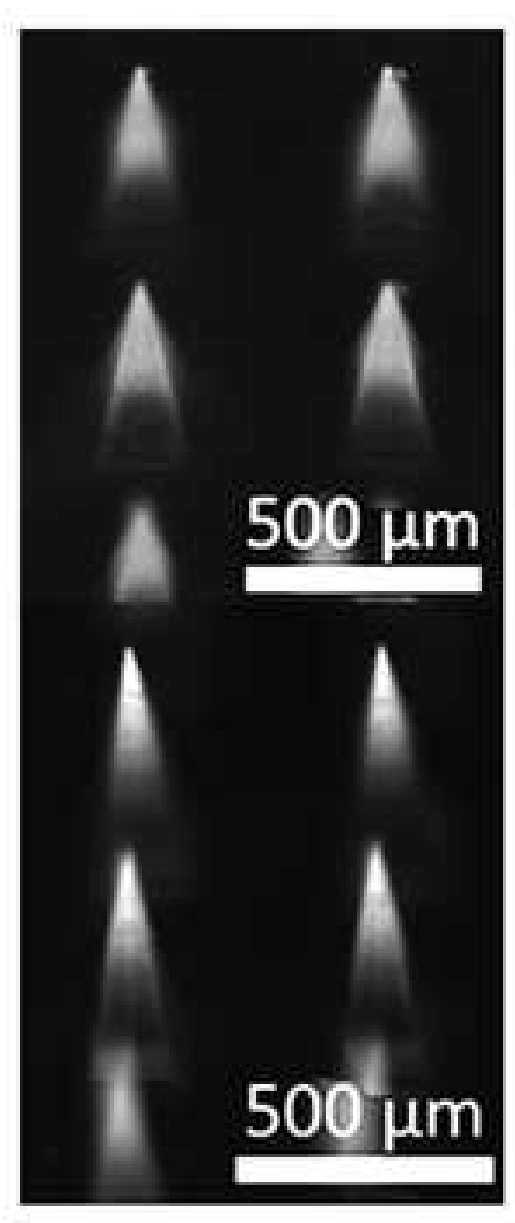

B

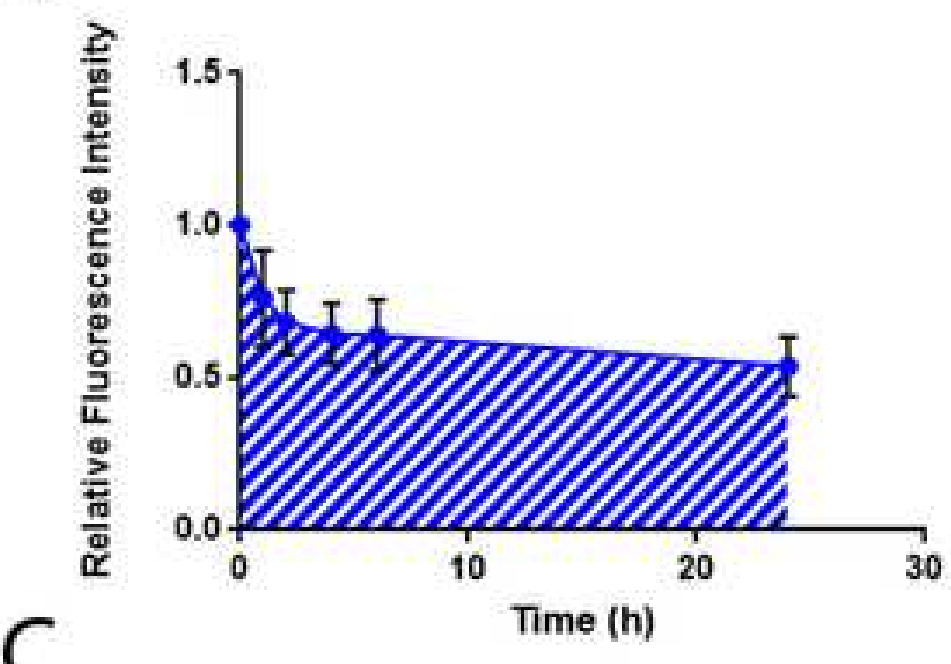

C

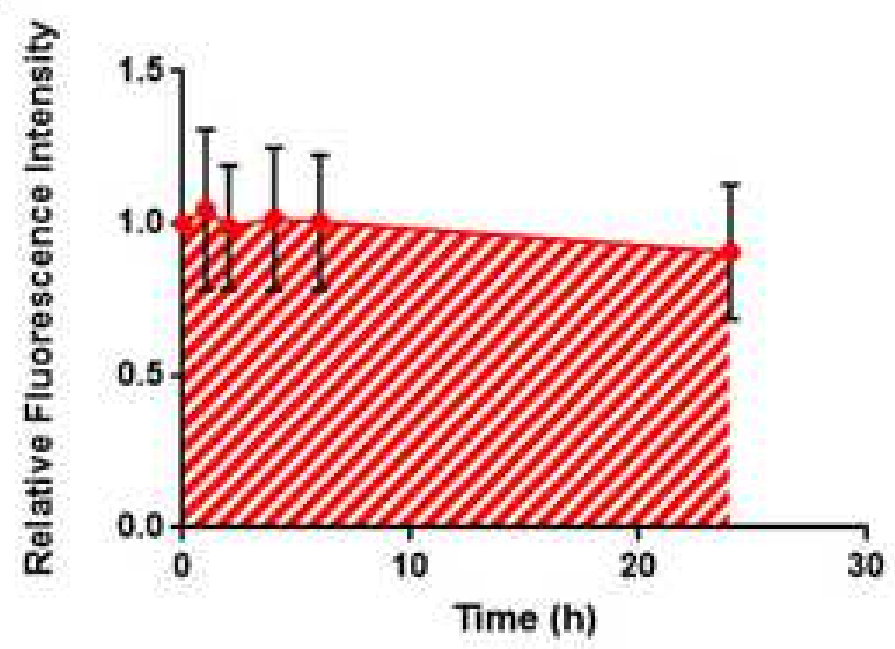

D

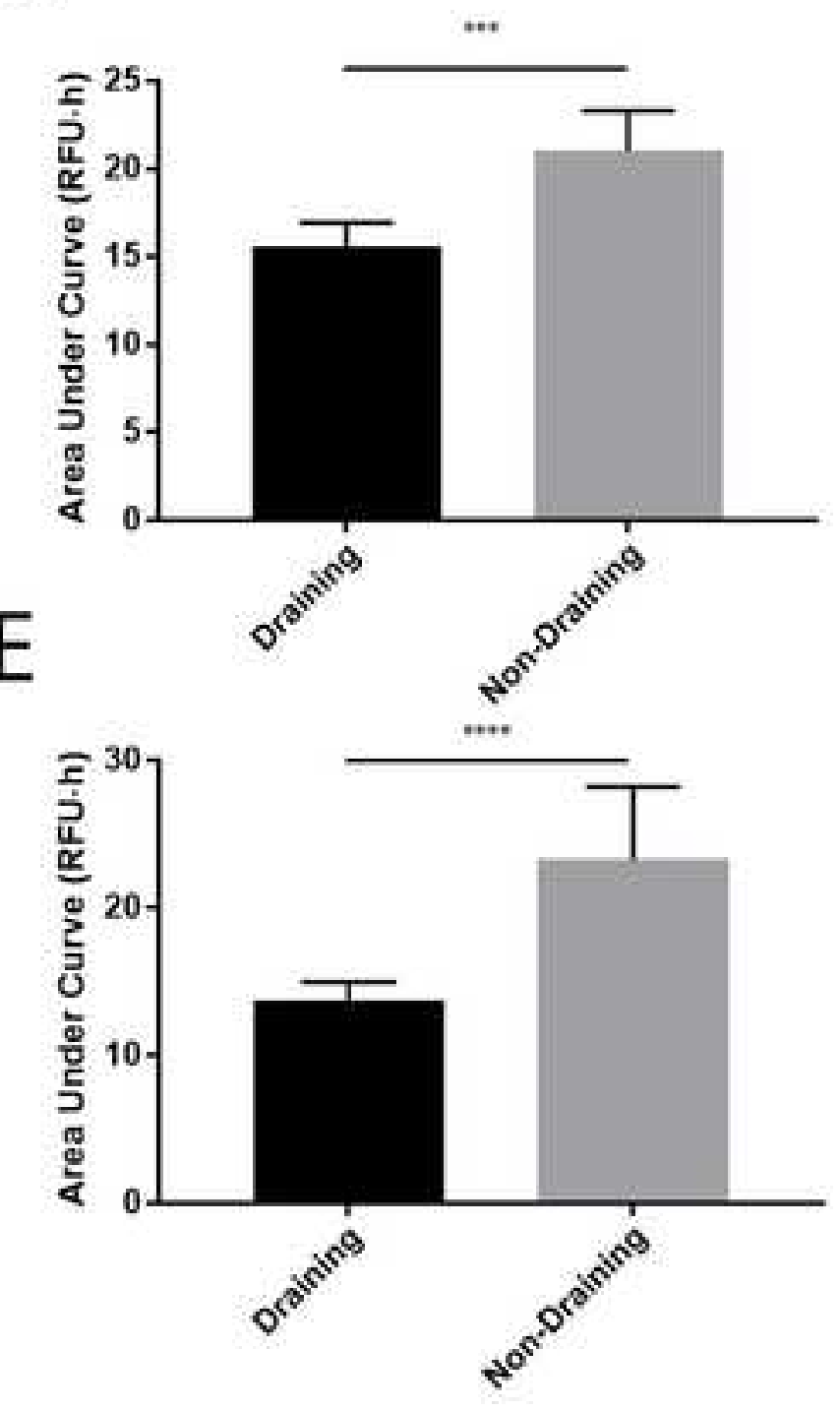


Supplementary Material
Click here to download Supplementary Material: Supporting Information_Revised_2.pdf

Click here to download Supplementary Material: Supporting Information Revised_apdf

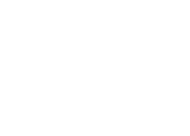

(1) (1) (1) (1) (1) (1)

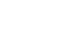
. . . . . . . . . . . . . . . . . . . . . . 